\title{
Carpooling and Congestion Pricing: HOV and HOT Lanes*
}

\author{
Hideo Konishi ${ }^{\dagger} \quad$ Se-il Mun $^{\ddagger}$
}

\begin{abstract}
It is often argued in the US that HOV (high occupancy vehicle) lanes are wasteful and should be converted to HOT (high occupancy vehicles and toll lanes). In this paper, we construct a simple model of commuters using a highway with multiple lanes, in which commuters are heterogeneous in their carpool organization costs. We first look at the HOV lanes and investigate under what conditions introducing HOV lanes is socially beneficial. Then we examine whether converting HOV lanes to HOT lanes improves the efficiency of road use. It is shown that the result depends on functional form and parameter values. We also discuss the effect of alternative policies: simple congestion pricing without lane division; and congestion pricing with HOV lanes. The analysis using specific functional form is presented to explicitly obtain the conditions determining the rankings of HOV, HOT, and other policies based on aggregate social cost.
\end{abstract}

\section{Introduction}

High occupancy vehicle (HOV) lanes have been introduced in many cities in the US and abroad in order to encourage commuters to carpool and ease traffic congestions. According to an analysis by the U.S. Census Bureau (2004), the share of people carpooling was 12.2 percent in 2000, which accounts for the second largest share: 77 percent of people drive alone; 4.7 percent use public transit. ${ }^{1}$ Recently, however, single occupancy vehicle drivers complain about underused HOV lanes, and many transportation researchers also criticize HOV lanes for their poor effectiveness in easing congestion. Employing a dynamic queuing model with

*Special thanks are due to Robin Lindsey for his helpful comments and encouragement. We are also grateful to the conference/seminar participants at PET 2008 in Seoul, RSAI 2008 in Brooklyn, Kyoto University, and University of Tokyo for their comments.

${ }^{\dagger}$ Department of Economics Boston College, 140 Commonwealth Ave. Chestnut Hill, MA 02467, USA.

${ }^{\ddagger}$ Graduate School of Economics, Kyoto University, Yoshida Hon-machi, Sakyo-ku, Kyoto 606-8501, Japan

${ }^{1}$ Note that the above figures are averages of the whole country: the share of carpooling should be higher in the areas where HOV lanes are available. For example, the share of carpooling is around $40 \%$ in Washington D.C. (see Table 7 of Houde, Safirova, and Harrington 2007). 
discrete choices, Dahlgren (1998) argues that adding a regular lane to existing lanes is more effective in reducing delay costs than adding an HOV lane in most cases. Yang and Huang (1999) also show that HOV lanes may reduce social welfare. Poole and Balaker (2005) report that recent evidence suggests that HOV lanes shift some travelers from vanpools and buses to less efficient carpools. Pointing out that $43 \%$ of carpoolers are members of the same household as some other carpooler, Fielding and Klein (1993) propose to convert HOV lanes to high occupancy and toll (HOT) lanes that are not only for carpoolers but also for solo drivers who are willing to pay tolls. HOT lanes have been adopted by the Los Angeles, San Diego, Houston, Salt Lake City, Denver, and Minneapolis-St. Paul metropolitan areas, and many other cities are considering introducing HOT lanes.

The reasons that HOT lanes are so hot are mainly three-fold: First, HOT lanes can make better use of underused HOV lanes, and ease the traffic in the regular lanes by shifting some drivers to HOT lanes. Second, HOT lanes generate revenue that can be used to finance new roads and lanes. Finally, HOT lanes are politically feasible policies. Although researchers agree that congestion pricing is the only policy that will make a noticeable difference in peak congestion (see Small, 1997), congestion pricing is regarded as politically infeasible. Obviously, political feasibility is important, and raising revenues from tolls is important, too. However, there is only a limited number of papers that analyze HOV and HOT lanes from a social welfare point of view. Safirova, Gillingham, Parry, Nelson, Harrington, and Mason (2004) compute the effects of converting existing HOV lanes to HOT Lanes for metropolitan Washington, D.C., and show that although all income groups, on aggregate, benefit from the policy, among them, wealthier households benefit considerably more than the lowestincome households. Small, Winston, and Yan (2006) show similar results by employing numerical simulations based on a general and empirically estimatable discrete-choice model. ${ }^{2}$ Although their results are based on a realistic model, their simulations are conducted for a limited range of parameter values. Therefore, it is still unclear whether their results generally hold. Yang and Huang (1999) considered combinations of congestion pricing and HOV lanes assuming that each commuter would incur an identical cost to form a carpool. However, they assume that the congestion toll is levied on both lanes; thus their results are not about HOT lanes. On the other hand, Small and Yan (2001) and Verhoef and Small (2004) analyze welfare performance of discriminatory pricing of different lanes assuming that commuters are heterogeneous in their time values. ${ }^{3}$ However, they do not consider endogenous carpools in their model. ${ }^{4}$ Thus, the welfare effects of policies associated with car-pooling remain largely unknown.

In this paper, we introduce a simple model with commuters whose carpool organization

\footnotetext{
${ }^{2}$ Although results are not shown in the main text, they suggest in the introduction that HOT lanes may lower social welfare compared with keeping all lanes regular, if they are priced high enough to allow motorists to travel at approximately free-flow speeds.

${ }^{3}$ In the literature of industrial organization, Reitman (1991) analyzed if a monopolist can improve its profit by using discriminatory pricing in a queueing model with heterogeneous time values.

${ }^{4}$ Small and Yan (2001) include car-pooling in their simulations to compute the effects of alternative pricing policies. However, the number of car-poolers is given exogenously and therefore not affected by different policies.
} 
costs are heterogeneous. This model is probably the simplest possible model that can analyze both HOV lanes and HOT lanes. For analytical tractability, we assume that commuters have the same time value, in order to focus on incentives to carpool. Obviously, assuming homogeneous time values has strong implications, and we are definitely assuming away some practical aspects. While HOV lanes can be seen as a sorting mechanism to sort out travelers with different carpool organization costs, HOT lanes can further sort out travelers with different congestion costs. Since we do not incorporate the latter effect with homogeneous time values, the result will have a bias against HOT lanes. ${ }^{5}$ When travelers' congestion costs are very different, introducing HOT lanes is more likely to improve the efficiency of HOV lanes. Of course, ultimately, the most important thing is to provide policy recommendations by taking practical and political considerations into account. However, we believe that it is also important to analyze a simple benchmark case fully to get a good sense of the relationships among the firstbest, secondbest, uniform congestion pricing, HOV, and HOT policies. In the literature, it is not even known how the firstbest policy looks like. As long as the implications of simplification assumption are correctly recognized, we believe that a complete analysis of simplified model will be quite useful to understand the realworld problem. ${ }^{6}$ We will provide cautious remarks on our analysis in the conclusion section.

The results of this paper are as follows. We first consider HOV and HOT policies in which the capacities of HOV/HOT lanes and regular lanes are predetermined, and no toll can be imposed on regular lane users. This is the closest case to the real-world HOV/HOT lanes, but the results really depend on the particular situation. As is shown in Dahlgren (1998), introducing HOV lanes can improve or deteriorate social welfare in our model. Converting HOV lanes to HOT lanes can improve or deteriorate social welfare, too. In some cases, converting HOV lanes to HOT lanes reduces every commuter's welfare for all positive tolls. We further discuss the effect of two alternative policies: uniform congestion pricing and differential pricing with lane division. Uniform pricing is equivalent to a conventional Pigouvian toll, but we show that this policy is not the first-best option. The optimal allocation can be attained by differential pricing in which there are two groups of lanes with differentiated tolls and no charge for carpoolers ${ }^{7}$. We characterize the optimal toll structures under differential pricing for two possibilities: solo drivers do not use HOV/HOT lanes; solo drivers use HOV/HOT lanes. Our results suggest that under the differential pricing, a positive toll needs to be charged on regular lanes even in the presence of HOV/HOT lanes. Optimal toll structure in the latter case may provide justification to HOT policy in that a higher toll is charged on HOV lanes. However, a (lower) toll on regular lanes is necessary to accompany a HOT policy, since otherwise the carpooling incentive is thwarted. This policy reduces the

\footnotetext{
${ }^{5}$ Verhoef and Small (2004) stressed the danger of underestimating such a bias by assuming heterogeneous time values away (in a model without carpooling possibilities). See also Small and Yan (2001).

${ }^{6}$ Allowing commuters' heterogeneity in their time values makes our model more realistic. We adopt homogeneous time values for analytical simplicity: otherwise, we need to deal with consumers' joint frequency distribution over time values and carpooling organization costs. It would be hard to get empirical estimates on such joint distribution.

${ }^{7}$ The differential pricing is essentially same as two-route HOT examined by Small, Winston, and Yan (2006).
} 
total number of vehicles by promoting carpooling while controlling the distortion from any difference in congestion levels between lanes.

This paper is organized as follows. In Section 2 we present a formal model. Section 3 provides an analysis of HOV and HOT lanes when regular lanes are free. In Sections 4, we discuss alternative pricing policies. Section 5 provides an example illustrating the analysis using specific functional forms. Section 6 concludes. Most proofs and examples are included in the appendices.

\section{The Model}

There is a highway that connects a suburb and the CBD (central business disctrict). All commuters must travel from the suburb to the CBD (inelastic demand). The highway has multiple lanes. These lanes are partitioned into two groups: $\alpha$ lanes and $\beta$ lanes. We normalize the total number of lanes to one, and describe the numbers (capacities) of $\alpha$ and $\beta$ lanes as fractions. The capacities of $\alpha$ and $\beta$ lanes are denoted by $K_{\alpha}$ and $K_{\beta}$, respectively $\left(K_{\alpha}+K_{\beta}=1\right)$. When we endogenize lane capacities, we ignore capacity indivisibility. Except for the case of uniform congestion pricing, we let carpoolers use $\alpha$ lanes if any: i.e., $\alpha$ lanes are used as HOV or HOT lanes. We assume that $m$ carpoolers share one car. ${ }^{8}$ The cost of organizing carpooling is heterogeneous (there are neighbors who have the same destination or not, or have small kids or not, etc.). Types of commuters are described by their carpool organization costs $t \in \mathbb{R}_{+}$. There is a unit mass of commuters with their $t$ distributed according to distribution function $F: \mathbb{R}_{+} \rightarrow[0,1]$ with density $f$. The measure of commuters who choose lane $i \in\{\alpha, \beta\}$ is denoted $n_{i} \geq 0$, and $n_{\alpha}+n_{\beta}=1$. The measure of cars (traffic volume) in lane $i \in\{\alpha, \beta\}$ is denoted by $Q_{i}$. The commuting cost itself is common to all drivers, and depends on the congestion ratio (volume-capacity ratio), $q_{i}$, i.e., $C\left(q_{i}\right)=C\left(Q_{i} / K_{i}\right)$.We assume that $C$ is twice continuously differentiable and convex, $C(q) \geq 0, C^{\prime}(q) \geq 0$ and $C^{\prime \prime}(q) \geq 0$ for all $q \geq 0$. We partition $\alpha$ lane users into two groups, carpoolers and solo drivers: $n_{\alpha}^{c p}+n_{\alpha}^{s}=n_{\alpha}$, where $n_{\alpha}^{c p}$ and $n_{\alpha}^{s}$ represent measures of $\alpha$ lane users who are carpoolers and solo drivers, respectively. A type $t$ commuter's total cost is described by

$$
C\left(q_{i}\right)+e t+\tau_{i}^{e}
$$

where $q_{i}$ is the volume-capacity ratio of lane $i \in\{\alpha, \beta\}$, and $e \in\{0,1\}$ denotes the commuter's carpooling decision: if no carpooling $e=0$ holds, if carpooling $e=1$ holds. The last term $\tau_{i}^{e}$ is the toll of type $i$ lanes when the commuter's carpooling decision is $e \in\{0,1\}$.

\footnotetext{
${ }^{8}$ In practice, the definition of a high occupancy vehicle is the one with $m$ people or more $(m=2,3$, or 4$)$. That is, some cars may carry more than $m$ people. Here, we assume that all HOVs carry exactly $m$ people. This is justified by interpreting that $m$ is the minimal passengers for being eligible to use HOV lanes. In this case, each commuter should choose $m$ to minimize the cost of carpooling.
} 


\section{HOV and HOT Lanes}

From now on, we assume that $K_{\alpha}$ and $K_{\beta}$ are fixed, and that $\alpha$ lanes are for commuters who use carpooling (HOV) and possibly for solo-drivers who pay a toll (HOT). In either case, $\beta$ lanes are free of charge. Thus, the problem is necessarily the second-best one. We first start with the case of complete laissez faire: no HOV lane and no toll. Then, we consider HOV lanes only. We investigate under what conditions introducing HOV lanes is socially beneficial.

\subsection{Without Policy Intervention}

Suppose that neither $\alpha$ nor $\beta$ is an HOV lane. Since there is no incentive to carpool, we have $Q_{\alpha}=n_{\alpha}$ and $Q_{\beta}=n_{\beta}$. Due to arbitrage, $Q_{\alpha} / K_{\alpha}=Q_{\beta} / K_{\beta}=1$. Thus, everybody pays the same commuting cost $C(1)$.

\subsection{With HOV Lane}

Suppose that lane $\alpha$ is now an HOV lane. Let type $\bar{t}$ be indifferent between the HOV lane $\alpha$ and the regular lane $\beta$. All drivers with $t \leq \bar{t}$ use the HOV lane, thus the number of commuters who use the HOV lane is $n_{\alpha}=F(\bar{t})$, which means that the number of cars on the HOV lane is $\frac{F(\bar{t})}{m}$, since $m$ people share the same car. Thus, $Q_{\alpha}=\frac{F(\bar{t})}{m}$. The conventional lane is used by $1-F(\bar{t})$ commuters, and $Q_{\beta}=1-F(\bar{t})$ (single person in each car). If type $\bar{t}$ commuter uses the HOV lane, the cost is

$$
C\left(q_{\alpha}\right)+\bar{t}=C\left(\frac{F(\bar{t})}{m K_{\alpha}}\right)+\bar{t}
$$

If type $\bar{t}$ commuter uses the regular lane, the cost is

$$
C\left(q_{\beta}\right)=C\left(\frac{1-F(\bar{t})}{K_{\beta}}\right)
$$

Since type $\bar{t}$ is indifferent between the two lanes, we have

$$
C\left(\frac{F(\bar{t})}{m K_{\alpha}}\right)+\bar{t}=C\left(\frac{1-F(\bar{t})}{K_{\beta}}\right) .
$$

Since $C$ is monotonically increasing, $C(0)<C\left(\frac{1}{K_{\beta}}\right)$ and $C\left(\frac{1}{m K_{\alpha}}\right)>C(0)$ hold. Thus the above equation has a unique solution: $\bar{t}^{H O V}$. The total social cost in equilibrium with HOV lanes is

$$
S C^{H O V}=F\left(\bar{t}^{H O V}\right) \times C\left(\frac{F\left(\bar{t}^{H O V}\right)}{m K_{\alpha}}\right)+\int_{0}^{\bar{t}^{H O V}} t d F+\left(1-F\left(\bar{t}^{H O V}\right)\right) \times C\left(\frac{1-F\left(\bar{t}^{H O V}\right)}{K_{\beta}}\right)
$$




\subsection{Are HOV Lanes Cost-Reducing?}

Proposition 1 Let $t_{\alpha}$ be a type that satisfies $F\left(t_{\alpha}\right)=K_{\alpha}$. Then, an introduction of HOV lane with capacity $K_{\alpha}$ reduces traffic on both $\mathrm{HOV}$ and regular lanes and is Pareto-improving if and only if

$$
C(1)-C\left(\frac{1}{m}\right) \geq t_{\alpha} .
$$

The condition says that if there are many low-carpool-organization-cost-type commuters, Pareto improvement can be achieved. We, however, provide a cautious remark on the above result. Our model does not consider "operation costs" of the car such as gasoline and parking costs. If they were included, low $t$ type commuters would carpool even if there were no HOV lane. The introduction of an HOV lane improves these commuters' commuting costs and encourages carpooling further. However, regular lanes can be more easily congested in this case, since low-carpool-organization-cost commuters would carpool even without HOV lanes. ${ }^{9}$

What about the aggregate social cost? If Pareto-improvement is made, the aggregate social cost is obviously improved. Thus, the above condition in the proposition is a sufficient condition for a social cost reduction. However, in some cases, introducing HOV lanes can deteriorate the social welfare by increasing the social cost. When $K_{\alpha}$ is too large, it is not surprising that introducing HOV lanes can deteriorate the social welfare. But this is not the only case. An example (Example 1) in the appendix shows that introducing HOV lanes can increase every commuter's cost even when $K_{\alpha}$ is reasonably low $\left(K_{\alpha}=1 / 4\right)$.

\subsection{HOT Lanes}

In O'Sullivan (2007), it is reported that the Riverside Freeway (California State Route 91) converted its two HOV lanes to HOT lanes (either high occupancy vehicles or toll-paying cars can use HOT lanes). O'Sullivan says, "The conversion to a HOT increases traffic volume, with about $80 \%$ of users paying the toll. The conversion also decreased traffic volume and increased speeds along the regular lanes on Route 91, generating benefits for other commuters" (page 218).

Here, we investigate O'Sullivan's statement through our simple model. Let $\tau>0$ be the toll for the HOT lanes. There are two types of users of HOT lanes: HOV users and solo drivers. Let the numbers of HOV users and solo drivers be $n_{\alpha}^{c p}$ and $n_{\alpha}^{s}$, respectively. Then, the HOV user $t$ 's incentive condition is

$$
C\left(\frac{n_{\alpha}^{c p}}{m K_{\alpha}}+\frac{n_{\alpha}^{s}}{K_{\alpha}}\right)+t \leq C\left(\frac{1-n_{\alpha}^{c p}-n_{\alpha}^{s}}{K_{\beta}}\right),
$$

the toll user t's incentive condition is

$$
C\left(\frac{n_{\alpha}^{c p}}{m K_{\alpha}}+\frac{n_{\alpha}^{s}}{K_{\alpha}}\right)+\tau \leq C\left(\frac{1-n_{\alpha}^{c p}-n_{\alpha}^{s}}{K_{\beta}}\right),
$$

\footnotetext{
${ }^{9}$ Note that this implies that our model has a bias against HOV lanes.
} 
and the regular lane users' incentive condition is

$$
C\left(\frac{n_{\alpha}^{c p}}{m K_{\alpha}}+\frac{n_{\alpha}^{s}}{K_{\alpha}}\right)+\tau \geq C\left(\frac{1-n_{\alpha}^{c p}-n_{\alpha}^{s}}{K_{\beta}}\right) .
$$

Thus, in equilibrium, the toll users and the regular lane users must be indifferent between the two types of lanes, i.e.,

$$
C\left(\frac{n_{\alpha}^{c p}}{m K_{\alpha}}+\frac{n_{\alpha}^{s}}{K_{\alpha}}\right)+\tau=C\left(\frac{1-n_{\alpha}^{c p}-n_{\alpha}^{s}}{K_{\beta}}\right) .
$$

Let us denote by $\bar{t}^{H O T}$ the threshold type of users so that the HOV users are type $t \leq \bar{t}^{H O T}$. Then equilibrium also requires

$$
C\left(\frac{n_{\alpha}^{c p}}{m K_{\alpha}}+\frac{n_{\alpha}^{s}}{K_{\alpha}}\right)+\bar{t}^{H O T}=C\left(\frac{1-n_{\alpha}^{c p}-n_{\alpha}^{s}}{K_{\beta}}\right) .
$$

Hence, the toll and regular lane users are type $t>\bar{t}^{H O T}$, and $\bar{t}^{H O T}=\tau$ holds. Since $n_{\alpha}^{c p}=F\left(\bar{t}^{H O T}\right)=F(\tau)$, the equilibrium with HOT lanes is characterized by

$$
C\left(\frac{F(\tau)}{m K_{\alpha}}+\frac{n_{\alpha}^{s}}{K_{\alpha}}\right)+\tau=C\left(\frac{1-F(\tau)-n_{\alpha}^{s}}{K_{\beta}}\right) .
$$

\subsection{Converting HOV Lanes to HOT Lanes}

Now, let us go back to the equilibrium with HOV lanes and consider converting HOV lanes into HOT lanes. In the HOV lane equilibrium, we have

$$
C\left(\frac{F\left(\bar{t}^{H O V}\right)}{m K_{\alpha}}\right)+\bar{t}^{H O V}=C\left(\frac{1-F\left(\bar{t}^{H O V}\right)}{K_{\beta}}\right) .
$$

and all type $t \leq \bar{t}^{\mathrm{HOV}}$ commuters choose HOV lanes while all $t>\bar{t}^{\mathrm{HOV}}$ commuters choose regular lanes. It is easy to see that if $\tau \geq \bar{t}^{H O V}$, conversion of HOV lanes to HOT lanes has no effect, since no commuters are willing to pay the toll. And if $\tau<\bar{t}^{H O V}$, then there would be a positive measure of toll users.

Let us conduct a comparative static analysis with respect to $\tau$ when $\tau \leq \bar{t}^{H O V}$. Totally differentiating (3), we have

$$
\frac{d n_{\alpha}^{s}}{d \tau}=\frac{-\left(\frac{C^{\prime}\left(\frac{F(\tau)}{m K_{\alpha}}+\frac{n_{\alpha}^{s}}{K_{\alpha}}\right) F^{\prime}(\tau)}{m K_{\alpha}}+\frac{C^{\prime}\left(\frac{1-F(\tau)-n_{\alpha}^{s}}{K_{\beta}}\right) F^{\prime}(\tau)}{K_{\beta}}+1\right)}{\frac{C^{\prime}\left(\frac{F(\tau)}{m K_{\alpha}}+\frac{n_{\alpha}^{s}}{K_{\alpha}}\right)}{K_{\alpha}}+\frac{C^{\prime}\left(\frac{1-F(\tau)-n_{\alpha}^{s}}{K_{\beta}}\right)}{K_{\beta}}}<0 .
$$

We now have the following proposition. 
Proposition 2 There is a toll $\tau\left(=\bar{t}^{H O V}\right)$ such that equilibrium with HOV lanes is equivalent to equilibrium with HOT lanes, where no commuter pays a toll. Starting from the HOT equilibrium with $\tau=\bar{t}^{H O V}$, reduce $\tau$ slightly. Then, the social welfare goes down if

$$
F^{\prime}(\tau)+\frac{d n_{\alpha}^{s}}{d \tau} \leq 0
$$

or

$$
F^{\prime}(\tau) \times C^{\prime}\left(\frac{F(\tau)}{m K_{\alpha}}\right) \leq K_{\alpha} \times \frac{m}{m-1},
$$

for $\tau \in\left(\bar{t}^{H O V}-\epsilon, \bar{t}^{H O V}\right)$ for small $\epsilon>0$. Thus, with some toll lower than $\bar{t}^{\mathrm{HOV}}$, converting HOV lanes to HOT lanes improves the social welfare under (5).

The above condition shows that if $K_{\alpha}$ is high and $m$ is low, then HOT lanes tend to dominate HOV lanes. This makes sense since high $K_{\alpha}$ and low $m$ make HOV lanes inefficient. However, it is a pretty strict requirement. Consider a uniformly distributed $F$ over interval $\left[0, \frac{1}{f}\right]$ (with density $F^{\prime}(t)=f$ for all $t \in\left[0, \frac{1}{f}\right]$ ), linear cost function $C(q)=c q$, and $K_{\alpha}=\frac{1}{4}$

and $m=4$. Then, the condition becomes $f c \leq \frac{1}{3}$. That is, the distribution of the carpool organization cost should not be very condensed and the congestion cost is relatively mild. Under this condition, HOT lanes are guaranteed to perform better than HOV lanes. Note, however, that the above condition is nothing but a sufficient condition. Even if it is violated, HOT lanes can perform better than HOV lanes. It appears to be widely believed that converting underused HOV lanes to HOT lanes is a good idea in order to reduce traffic in the conventional lane. On the other hand, however, if the traffic in the HOV lane increases, that might discourage commuters from organizing a carpool despite the original objective of introducing HOV lanes. An example (Example 2) in the appendix shows that the latter negative effect may dominate the former positive effect in a strong manner (in the Pareto sense), and that HOT lanes perform worse than HOV lanes.

\section{Alternative Pricing Policies}

Under HOV or HOT policy, regular lane users could not be charged a toll. In this section, we remove this restriction, and analyze alternative policies. We also allow the transit authority to choose $K_{\alpha}$.

\subsection{Uniform Congestion Pricing}

Suppose that there is no division of lanes on the highway and the transportation authority chooses the toll that minimizes the social cost. In this case, commuting costs in all lanes of the highway must be the same. Since the number of commuters is fixed, the sole role of tolling is to control the level of carpooling, which is represented by the threshold value $\bar{t}$ 
commuter type: all commuters with $t \leq \bar{t}$ carpool, but not others. The social aggregated cost in such an allocation characterized by $\bar{t}$ is

$$
C\left(1-F(\bar{t})+\frac{F(\bar{t})}{m}\right) \times 1+\int_{0}^{\bar{t}} t f(t) d t .
$$

The first-order condition with respect to $\bar{t}$ for the minimization of the above (assuming an interior solution) is

$$
C^{\prime}\left(1-F(\bar{t})+\frac{F(\bar{t})}{m}\right)\left(-f(\bar{t})+\frac{f(\bar{t})}{m}\right)+\bar{t} f(\bar{t})=0,
$$

or

$$
\frac{m-1}{m} C^{\prime}\left(1-F(\bar{t})+\frac{F(\bar{t})}{m}\right)=\bar{t} .
$$

The LHS shows the commuting cost savings from increasing $\bar{t}$, while the RHS shows the organizing cost increase from increasing $\bar{t}$, and the first-order condition shows that these two are equated. Let us denote by $\bar{t}^{U}$ the solution to the above equation.

We derive the uniform toll to attain $\bar{t}^{U}$ in a decentralized equilibrium. If a type $\bar{t}^{U}$ commuter carpools, the cost she pays is

$$
C\left(1-F\left(\bar{t}^{U}\right)+\frac{F\left(\bar{t}^{U}\right)}{m}\right)+\bar{t}^{U}+\frac{\tau}{m},
$$

and if she does not, she pays

$$
C\left(1-F\left(\bar{t}^{U}\right)+\frac{F\left(\bar{t}^{U}\right)}{m}\right)+\tau .
$$

In equilibrium, type $\bar{t}^{U}$ commuters need to be indifferent between paying toll $\tau^{U}$ and carpooling. Thus, the optimal uniform toll $\tau^{U}$ to attain $\bar{t}^{U}$ is

$$
\tau^{U}=\frac{m}{m-1} \bar{t}^{U}=C^{\prime}\left(1-F\left(\bar{t}^{U}\right)+\frac{F\left(\bar{t}^{U}\right)}{m}\right) .
$$

The latter equality holds by the first-order condition (7). The above pricing rule is equivalent to the conventional Pigouvian toll: the toll should be equal to the congestion externality that is the sum of the delay caused by an additional vehicle for all road users.

There is another way to achieve the same allocation as above if $K_{\alpha}$ and $K_{\beta}$ can be chosen freely. Let us assume that $\alpha$ lanes are HOV lanes that only carpoolers use. Thus the number of cars for $\alpha$ lanes is $\frac{F\left(\bar{t}^{U}\right)}{m}$, and the one for $\beta$ lanes is $1-F\left(\bar{t}^{U}\right)$. Since capacities should be chosen so that the congestion level is common to all lanes, we have

$$
K_{\alpha}^{*}=\frac{\frac{F\left(\bar{t}^{U}\right)}{m}}{\frac{F\left(\bar{t}^{U}\right)}{m}+1-F\left(\bar{t}^{U}\right)}=\frac{F\left(\bar{t}^{U}\right)}{m-(m-1) F\left(\bar{t}^{U}\right)} .
$$


To decentralize this allocation, $\beta$ lane should be tolled. Suppose that $\beta$ lanes charge a toll $\tau_{\beta}^{*}$. If a type $\bar{t}^{U}$ commuter chooses an $\alpha$ lane, she pays

$$
C\left(1-F\left(\bar{t}^{U}\right)+\frac{F\left(\bar{t}^{U}\right)}{m}\right)+\bar{t}^{U},
$$

and if she chooses a $\beta$ lane, she pays

$$
C\left(1-F\left(\bar{t}^{U}\right)+\frac{F\left(\bar{t}^{U}\right)}{m}\right)+\tau_{\beta}^{*} .
$$

Hence, we have

$$
\tau_{\beta}^{*}=\bar{t}^{U}
$$

This is summarized as the following proposition.

Proposition 3 The allocation under the optimal uniform pricing can be achieved by using HOV lanes with capacity

$$
K_{\alpha}^{*}=\frac{F\left(\bar{t}^{U}\right)}{m-(m-1) F\left(\bar{t}^{U}\right)},
$$

and by charging regular lane commuters a discriminatory toll

$$
\tau_{\beta}^{*}=\bar{t}^{U} .
$$

There are three important points on this decentralization. First, if HOV lanes are used, their capacity needs to be chosen optimally. This means that if the number of HOV lanes cannot be chosen freely (by the integer problem or political considerations), the allocation with the optimal uniform toll cannot be achieved. Second, the toll is levied on non-HOV lanes. Third, both regular and HOV lanes have the same congestion level. Finally, we have $\tau^{U}=\frac{m}{m-1} \tau_{\beta}^{*}>\tau_{\beta}^{*}$. If there is political pressure to keep a toll low, the latter method may be more appealing.

It is important to realize that uniform congestion pricing is not the first-best policy. In the next section, we will see that the HOV lane policy can be better than the optimal uniform congestion pricing for some parameter range. Here, however, we will demonstrate that there is always a policy that is better than uniform congestion pricing. In order to show this, we utilize the above proposition. The optimal uniform pricing policy is equivalent to an HOV lane policy with capacity $K_{\alpha}^{*}$ and a regular lane toll $\tau_{\beta}^{*}$. The type $\bar{t}^{U}$ is indifferent between the two lanes. Now reduce $\tau$ so that the indifferent type $\bar{t}=\tau$ is reduced. From the previous subsection, we know ${ }^{10}$

$$
\begin{aligned}
\frac{d S C(\bar{t})}{d \bar{t}}= & \underbrace{C^{\prime}\left(\frac{F(\bar{t})}{m K_{\alpha}}\right) \frac{F(\bar{t})}{m K_{\alpha}}+C\left(\frac{F(\bar{t})}{m K_{\alpha}}\right)}_{\Delta S C \text { in HOV lanes by having more carpoolers }}+\underbrace{\bar{t}}_{\Delta \text { (carpooling costs) }} \\
& \underbrace{C^{\prime}\left(\frac{1-F(\bar{t})}{K_{\beta}}\right) \frac{(1-F(\bar{t}))}{K_{\beta}}-C\left(\frac{1-F(\bar{t})}{K_{\beta}}\right)}_{-\Delta S C \text { in regular lanes by having more carpoolers }} .
\end{aligned}
$$

${ }^{10}$ Note that equation $(10)$ is the same as $\frac{d S C(\bar{t})}{d \bar{t}}=0$. Setting $n_{\alpha}^{s}=0$, we obtain the formula here. 
Note that under uniform congestion pricing, the congestion levels of HOV and regular lanes are the same: i.e.,

$$
\frac{F\left(\bar{t}^{U}\right)}{m K_{\alpha}^{*}}=\frac{1-F\left(\bar{t}^{U}\right)}{K_{\beta}^{*}} .
$$

Thus, we have

$$
\left.\frac{d S C(\bar{t})}{d \bar{t}}\right|_{\bar{t}=\bar{t}^{U}}=\bar{t}^{U}>0 .
$$

This means that starting from the optimal uniform pricing allocation, we can reduce social costs by discouraging carpooling (by reducing $\tau=\bar{t}$ ). Such a policy obviously reduces traffic in HOV lanes and increases it in regular lanes. Therefore, we can conclude that under the optimal uniform congestion pricing, the carpooling level is too high in comparison with the efficient level.

\subsection{The Optimal Allocation with Arbitrary or Optimal Lane Di- vision}

As shown above, conventional Pigouvian toll is not efficient when the decision to carpool is involved. So what does the optimal policy look like? We first characterize the first best optimal allocation, then show that the optimal allocation can be attained in two lane setting where the road is divided into HOV (HOT) and regular lanes. Suppose that there are $n$ equally-sized lanes. That is, each lane has capacity $\frac{1}{n}$. The first-best is defined as the allocation of car-poolers and solo-drivers among $n$ lanes that minimizes the social cost, the sum of trip time cost and car-pooling organization cost. Details of optimality conditions are given in Appendix B. The optimal allocation is decentralized by levying the tolls per vehicle based on congestion externality. Thus tolls on k-th lane for solo-drivers and car-poolers are, $C^{\prime}\left(n Q_{k}\right) n N_{k}$ and $\frac{1}{m} C^{\prime}\left(n Q_{k}\right) n N_{k}$, respectively, where $Q_{k}$ and $N_{k}$ are the measures of traffic and users on lane $k$, respectively.

Should we levy differential toll on each of $n$ lanes? Clearly, $n$ lanes will be classified into three types of lanes: car-pool lanes, mixed lanes, and solo lanes.

Proposition 4 In the first-best allocation, we have

1. there exists $\bar{t} \in[0,1]$ such that the sum of carpoolers in car-pool lanes and mixed lanes is equal to $F(\bar{t})$.

2. (i) in all car-pool lanes and all mixed lanes, the level of congestion is the same, and (ii) in all solo lanes, the levels of congestion is the same.

This proposition has an interesting implication. At the first best allocation, commuters sort out by their car-pool organization cost $t$, and all car poolers experience the same congestion level, and all solo drivers also experience the same congestion level. Even with a 
large number of lanes, there are only two congestion levels. So $n$ lanes can be bundled into two groups of lanes, in each of which congestion level is the same. This implies that we can rewrite the optimization problem for $n$ lanes as the one for two types of lanes, i.e., in a form compatible to that presented in the previous sections. Let fraction $K_{\alpha}=\frac{\bar{k}}{n}$ lanes be reserved for HOV (or HOT) lanes ( $\alpha$ lanes), and fraction $K_{\beta}=\frac{n-\bar{k}}{n}$ lanes for solo lanes ( $\beta$ lanes), where $\bar{k}$ is the sum of car-pool and mix lanes.

Below we discuss the optimal allocation in two steps. First, we characterize the optimal allocation under an arbitrary lane division (i.e., allocation of lane capacity, $K_{\alpha}$ ). Second, among these optimal allocations under different lane divisions, we choose the lane division that minimizes the social cost: this is the first best allocation ${ }^{11}$.

In the first step, the optimization problem is basically to (i) choose $\bar{t} \in[0,1]$ such that all types $t \leq \bar{t}$ choose $\alpha$ lanes while all types $t>\bar{t}$ choose $\beta$ lanes, and (ii) choose $n_{\alpha}^{s}$ under the constraint of $0 \leq n_{\alpha}^{s}$. Since the optimal $\bar{t}$ must be an interior solution, the Kuhn-Tucker problem for the social cost minimization is:

$$
\begin{aligned}
L\left(\bar{t}, n_{\alpha}^{s}\right)= & {\left[C\left(\frac{F(\bar{t})}{m K_{\alpha}}+\frac{n_{\alpha}^{s}}{K_{\alpha}}\right) F(\bar{t})+\int_{0}^{\bar{t}} t d F+n_{\alpha}^{s} C\left(\frac{F(\bar{t})}{m K_{\alpha}}+\frac{n_{\alpha}^{s}}{K_{\alpha}}\right)\right.} \\
& \left.+\left(1-F(\bar{t})-n_{\alpha}^{s}\right) C\left(\frac{1-F(\bar{t})-n_{\alpha}^{s}}{K_{\beta}}\right)\right]-\lambda n_{\alpha}^{s} .
\end{aligned}
$$

The first-order conditions with respect to $\bar{t}$ and $n_{\alpha}^{s}$ are:

$$
\begin{aligned}
& C^{\prime}\left(\frac{F(\bar{t})}{m K_{\alpha}}+\frac{n_{\alpha}^{s}}{K_{\alpha}}\right) \frac{\left(F(\bar{t})+n_{\alpha}^{s}\right)}{m K_{\alpha}}+C\left(\frac{F(\bar{t})}{m K_{\alpha}}+\frac{n_{\alpha}^{s}}{K_{\alpha}}\right)+\bar{t} \\
& -C^{\prime}\left(\frac{1-F(\bar{t})-n_{\alpha}^{s}}{K_{\beta}}\right) \frac{\left(1-F(\bar{t})-n_{\alpha}^{s}\right)}{K_{\beta}}-C\left(\frac{1-F(\bar{t})-n_{\alpha}^{s}}{K_{\beta}}\right) \\
= & 0,
\end{aligned}
$$

and

$$
\begin{aligned}
& C^{\prime}\left(\frac{F(\bar{t})}{m K_{\alpha}}+\frac{n_{\alpha}^{s}}{K_{\alpha}}\right) \frac{\left(F(\bar{t})+n_{\alpha}^{s}\right)}{K_{\alpha}}+C\left(\frac{F(\bar{t})}{m K_{\alpha}}+\frac{n_{\alpha}^{s}}{K_{\alpha}}\right) \\
& -C\left(\frac{1-F(\bar{t})-n_{\alpha}^{s}}{K_{\beta}}\right)-C^{\prime}\left(\frac{1-F(\bar{t})-n_{\alpha}^{s}}{K_{\beta}}\right) \frac{\left(1-F(\bar{t})-n_{\alpha}^{s}\right)}{K_{\beta}}-\lambda \\
= & 0,
\end{aligned}
$$

respectively, where we have $\lambda \geq 0$ and $\lambda n_{\alpha}^{s}=0$.

We will show that the optimal allocation is decentralizable with a differential toll system. By arranging the optimality conditions, we obtain the following result.

\footnotetext{
${ }^{11}$ Choice of lane division does not exist in the problem for $n$ lanes: drivers sort themselves into solo lanes, car-pool lanes and mixed lanes, led by pricing on each of $n$ lanes.
} 
Proposition 5 In the optimal allocation under an arbitrary lane division, $\alpha$ lanes are always less crowded than $\beta$ lanes:

$$
\frac{F\left(\bar{t}^{D}\right)}{m K_{\alpha}}+\frac{n_{\alpha}^{s}}{K_{\alpha}}<\frac{1-F\left(\bar{t}^{D}\right)-n_{\alpha}^{s}}{K_{\beta}},
$$

where $\bar{t}^{D}$ and $n_{\alpha}^{s}$ are solutions of equations (10) and (11). The optimal allocation is decentralizable by charging differential tolls.(i) When $n_{\alpha}^{s}=0$, the optimal toll for solo-drivers on $\beta$ lanes is

$$
\tau_{\beta}^{D}=C^{\prime}\left(\frac{1-F\left(\bar{t}^{D}\right)}{K_{\beta}}\right) \frac{1-F\left(\bar{t}^{D}\right)}{K_{\beta}}-C^{\prime}\left(\frac{F\left(\bar{t}^{D}\right)}{m K_{\alpha}}\right) \frac{F\left(\bar{t}^{D}\right)}{m K_{\alpha}}>0 .
$$

(ii) When $n_{\alpha}^{s}>0$, the optimal toll for solo-drivers on $\beta$ lanes is

$$
\tau_{\beta}^{D}=C^{\prime}\left(\frac{1-F\left(\bar{t}^{D}\right)-n_{\alpha}^{s}}{K_{\beta}}\right)\left(\frac{1-F\left(\bar{t}^{D}\right)-n_{\alpha}^{s}}{K_{\beta}}\right)-C^{\prime}\left(\frac{F\left(\bar{t}^{D}\right)}{m K_{\alpha}}+\frac{n_{\alpha}^{s}}{K_{\alpha}}\right)\left(\frac{F\left(\bar{t}^{D}\right)+n_{\alpha}^{s}}{m K_{\alpha}}\right)>0,
$$

and the toll for solo-drivers on $\alpha$ lanes is higher than the one on $\beta$ lanes

$$
\begin{aligned}
\tau_{\alpha}^{D} & =C^{\prime}\left(\frac{F\left(\bar{t}^{D}\right)}{m K_{\alpha}}+\frac{n_{\alpha}^{s}}{K_{\alpha}}\right)\left(\frac{F\left(\bar{t}^{D}\right)+n_{\alpha}^{s}}{K_{\alpha}}\right)-C^{\prime}\left(\frac{F\left(\bar{t}^{D}\right)}{m K_{\alpha}}+\frac{n_{\alpha}^{s}}{K_{\alpha}}\right)\left(\frac{F\left(\bar{t}^{D}\right)+n_{\alpha}^{s}}{m K_{\alpha}}\right) \\
& =\tau_{\beta}^{D}+C\left(\frac{1-F\left(\bar{t}^{D}\right)-n_{\alpha}^{s}}{K_{\beta}}\right)-C\left(\frac{F\left(\bar{t}^{D}\right)}{m K_{\alpha}}+\frac{n_{\alpha}^{s}}{K_{\alpha}}\right)>\tau_{\beta}^{D} .
\end{aligned}
$$

The above pricing rule is equivalent to the first-best one while carpoolers are free of charge $^{12}$. Since $\beta$ lanes are tolled in the optimal allocation under any arbitrary lane division, simple HOV or HOT lane policy cannot support the optimal allocation. Tolling on $\beta$ lane gives commuters greater incentive to carpool. In this way, the optimal allocation can improve on simple HOV or HOT lane policy.

Clearly, the same statement holds when the division of lanes (choice of $K_{\alpha}$ ) is optimal.

Corollary 6 In the first-best allocation, $\alpha$ lanes are always less crowded than $\beta$ lanes, and the first-best allocation is decentralizable by charging different tolls provided in the previous proposition.

It may be somewhat interesting to consider the limit case: a large number of $n$ or a perfectly divisible lanes. Obviously, such a case is not realistic situation, but we can learn what sort of allocation realizes when the ideal lane division happens to be achievable with a finite lane case. We have the following lemma.

Lemma 7 In the first-best allocation, there is at most one mixed lane.

\footnotetext{
${ }^{12}$ Since total mass of commuters is fixed, the choice of commuters are affected only by the relative amount of tolls. The pricing rule here is obtained by subtracting the congestion externality by carpoolers.
} 
The above lemma immediately implies that as $n$ goes to infinity, the fraction of commuters who use the mixed lane becomes negligible, or $n_{\alpha}^{s}$ goes to zero. Thus, in the ideal situation, we have the following proposition.

Proposition 8 Suppose that $n$ goes to infinity, or that the globally optimal lane division $K_{\alpha}^{*}$ is achievable. Then, $n_{\alpha}^{s}=0$ holds.

The above proposition implies that the case $n_{\alpha}^{s}>0$ (as in HOT lanes) is not optimal in the ideal world, but arises as the result of indivisibility (the integer problem), or inefficient capacity allocation.

\section{A Special Case}

\subsection{Aggregate Social Costs under HOV and HOT lanes}

Let $F$ be uniform distribution over $[0,1]$ : i.e., $F(t)=t$ for $0 \leq t \leq 1$, and $C(q)=c q$ with $c>0$. If there is no HOV or HOT lane, then everybody pays $C(1)=c$; thus the aggregate social cost with no policy is $S C^{\emptyset}=c$. If there are HOV lanes, then an indifferent commuter $\bar{t}$ satisfies

$$
C_{\alpha}^{H O V}+\bar{t}=\frac{c \bar{t}}{m K_{\alpha}}+\bar{t}=\frac{c(1-\bar{t})}{1-K_{\alpha}}=C_{\beta}^{H O V}
$$

Solving the above equation yields

$$
\bar{t}^{H O V}=\frac{c m K_{\alpha}}{c m K_{\alpha}+\left(c+m K_{\alpha}\right)\left(1-K_{\alpha}\right)} .
$$

The aggregate social cost is

$$
\begin{aligned}
& S C^{H O V} \\
= & \bar{t}^{H O V} \times C_{\alpha}^{H O V}+\int_{0}^{\bar{t}^{H O V}} t d t+\left(1-\bar{t}^{H O V}\right) \times C_{\beta}^{H O V} \\
= & C_{\alpha}^{H O V}+\bar{t}^{H O V}+\int_{0}^{\bar{t} H O V}\left(t-\bar{t}^{H O V}\right) d t \\
= & \frac{c \bar{t}^{H O V}}{m K_{\alpha}}+\bar{t}^{H O V}-\frac{\left(\bar{t}^{H O V}\right)^{2}}{2} \\
= & \frac{c\left(c-m K_{\alpha}\right)}{c-c K_{\alpha}+c m K_{\alpha}+m K_{\alpha}-m K_{\alpha}^{2}}-\frac{1}{2}\left(\frac{c m K_{\alpha}}{c-c K_{\alpha}+c m K_{\alpha}+m K_{\alpha}-m K_{\alpha}^{2}}\right)^{2} .
\end{aligned}
$$

Thus, we have the following proposition. 
Proposition 9 The introduction of HOV lanes improves social welfare if and only if $c>$ $\tilde{c}\left(K_{\alpha}, m\right)$, where

$$
\begin{aligned}
& \widetilde{c}\left(K_{\alpha}, m\right) \\
= & \frac{4 K_{\alpha}^{2} m^{2}-3 K_{\alpha} m^{2}-4 K_{\alpha}^{2} m+4 K_{\alpha} m+\sqrt{-8 K_{\alpha}^{3} m^{4}+9 K_{\alpha}^{2} m^{4}+8 K_{\alpha}^{3} m^{3}-8 K_{\alpha}^{2} m^{3}}(14)}{2\left(2 K_{\alpha} m^{2}-4 K_{\alpha} m+2 m+2 K_{\alpha}-2\right)} \\
= & \frac{-m\left(3-4 K_{\alpha}\right)+4\left(1-K_{\alpha}\right)+\sqrt{m^{2}\left(9-8 K_{\alpha}\right)-8 m\left(1-K_{\alpha}\right)}}{4\left(m-2+\frac{1}{K_{\alpha}}+\frac{1}{m}-\frac{1}{K_{\alpha} m}\right)}
\end{aligned}
$$

The above proposition states that the introduction of HOV lanes improves the social welfare as the congestion cost exceeds a threshold level, which is a highly nonlinear function of $K_{\alpha}$ and $m$. Although it is difficult to directly deal with the inequality, we can say that HOV lanes policy is more likely to improve the social welfare as capacity of HOV lanes is smaller, or the number of people sharing the car is larger ${ }^{13}$.

Now, we turn to HOT lanes. Equilibrium with the HOT policy requires

$$
C_{\alpha}^{H O T}(\tau)+\tau=c\left(\frac{\tau}{m K_{\alpha}}+\frac{n_{\alpha}^{s}}{K_{\alpha}}\right)+\tau=c\left(\frac{1-\tau-n_{\alpha}^{s}}{1-K_{\alpha}}\right)=C_{\beta}^{H O T}(\tau),
$$

Solving the above equation yields

$$
n_{\alpha}^{s}=\frac{c m K_{\alpha}+\tau\left(m K_{\alpha}^{2}+c K_{\alpha}-c m K_{\alpha}-m K_{\alpha}-c\right)}{c m} .
$$

Thus,

$$
\begin{aligned}
C_{\beta}^{H O T}(\tau) & =c\left(\frac{1-\tau-n_{\alpha}^{s}}{1-K_{\alpha}}\right) \\
& =\frac{c m+\tau\left(-m c+c+m K_{\alpha}\right)}{m}
\end{aligned}
$$

The aggregate social cost under HOT is obtained as

$$
\begin{aligned}
& S C^{H O T}(\tau) \\
= & 1 \times C_{\beta}^{H O T}(\tau)+\underbrace{\int_{0}^{\tau}(t-\tau) d t}_{\begin{array}{c}
\text { cost saving for } \\
\text { HOV users }
\end{array}}-\underbrace{\tau \times n_{\alpha}^{s}}_{\text {toll revenue }} \\
= & \frac{\left(-2 m K_{\alpha}^{2}+2(c(m-1)+m) K_{\alpha}+c(2-m)\right) \tau^{2}+\left(2 c^{2}-2 c^{2} m\right) \tau+2 m c^{2}}{2 c m}
\end{aligned}
$$

\footnotetext{
${ }^{13}$ It is seen that $\widetilde{c}\left(K_{\alpha}, m\right)=0$ at $K_{\alpha}=0$ and $\widetilde{c}\left(K_{\alpha}, m\right)=\frac{m}{2(m-1)}$ at $K_{\alpha}=1$. This suggests that the threshold value $\widetilde{c}$ is increasing with $K_{\alpha}$, at least somewhere for $0<K_{\alpha} \leq 1$. As for the effect of $m$, the condition that HOV lanes policy reduces the social cost is expressed as $m>\widetilde{m}\left(c, K_{\alpha}\right)$.
} 
Differentiating $S C^{H O T}(\tau)$ with respect to $\tau$ and applying $\tau=0$, we have $\left.\frac{d S C^{H O T}(\tau)}{d \tau}\right|_{\tau=0}=$ $\frac{c(1-m)}{m}<0$ (see Appendix). We have the following result.

Proposition 10 Converting HOV lanes to HOT lanes (with some toll rate) improves the social welfare if and only if the following condition holds

$$
c<\frac{m K_{\alpha}}{m-1}
$$

Proposition 10 implies that converting HOV lanes to HOT lanes improves the social welfare, if (i) the unit cost of congestion, $c$, is small, (ii) $m$ is small, and (iii) $K_{\alpha}$ is large. Condition (ii) means that an SOV (single-occupant vehicle) does not impose much more congestion than does an HOV (say, if $m=2$ ). ${ }^{14}$ Condition (iii) means that if $\alpha$ lanes has large capacity, the social cost of underused $\alpha$ lanes is high. Converting HOV lanes to HOT lanes reduces congestion in $\beta$ lanes under such circumstances.

It should be noted that, in this special case, the sufficient condition in Proposition 3 becomes $c<\frac{m K_{\alpha}}{m-1}$. In other words, this inequality becomes a necessary and sufficient condition. The threshold relation $c=\frac{m K_{\alpha}}{m-1}$ is also critical for other results. Applying specifications to Proposition 1, the condition of Pareto improvement becomes $c>\frac{m K_{\alpha}}{m-1}$.

Combining the results so far, we classify the possible patterns regarding the effects of introducing HOV lanes and converting HOV lanes to HOT lanes, as depicted in Figure 1AC. ${ }^{15}$ The parameter range of each pattern is shown in Figure 2 in which letters A, B, C attached to areas correspond to the patterns in Figure 1 A, B, C, respectively. According to Figure 2, an HOV lanes policy is wasteful (the case of Figure $1 \mathrm{~A}$ emerges) when $K_{\alpha}$ is relatively large. This condition is consistent with the claim based on casual observations: in this case, HOV lanes are likely to be underused. It is also true that converting HOV to HOT lanes is not always effective for congestion mitigation: it improves the social welfare only if the capacity of the HOT lanes is larger and the congestion level is not too heavy (Figure 1A and $1 \mathrm{~B}$ ). There are situations where adopting simple HOV lanes is the best policy (Figure 1C). On the other hand, HOT lanes may be effective even when the introduction of HOV lanes aggravates the situation (Figure 1A).

In the cases of Figure $1 \mathrm{~A}$ and $\mathrm{B}$, there exists an optimal HOT toll that minimizes the aggregate social cost. Let us denote the optimal HOT toll by $\tau^{H O T}$, which is a solution of $\frac{d S C^{H O T}(\tau)}{d \tau}=0$. We have $\tau^{H O T}$ and the minimized social cost, $S C^{H O T}\left(\tau^{H O T}\right)$, as follows.

$$
\begin{aligned}
\tau^{H O T} & =\frac{c^{2}(m-1)}{-2 m K_{\alpha}^{2}+2(c(m-1)+m) K_{\alpha}-c(m-2)} \\
S C^{H O T}\left(\tau^{H O T}\right) & =-\frac{c\left(4 m^{2} K_{\alpha}^{2}-4 m(c(m-1)+m) K_{\alpha}+c\left(c(m-1)^{2}+2(m-2) m\right)\right.}{2 m\left(-2 m K_{\alpha}^{2}+2(c(m-1)+m) K_{\alpha}-c(m-2)\right)}(18)
\end{aligned}
$$

\footnotetext{
${ }^{14} \mathrm{It}$ is because in our model the carpool organization cost is assumed to be independent of the value of $m$.

${ }^{15}$ Although the curve in Figure $1 \mathrm{C}$ is concave, it may be convex in some cases, as discussed in the proof of Proposition 6.
} 
In the cases shown in Figure 1C, optimal policy is not to adopt HOT. Thus $S C^{H O T}\left(\tau^{H O T}\right)=$ $S C^{H O V}$ holds.

\subsection{Comparing Alternative Policies}

Under the uniform pricing policy, the number of HOV users is obtained by solving (8) as $\bar{t}^{U}=c \frac{m-1}{m}$. Substituting this in (6) we have the aggregate social cost as

$$
S C^{U}=-\frac{c\left(c(m-1)^{2}-2 m^{2}\right)}{2 m^{2}}
$$

There are two cases in the optimal pricing in Proposition 6: $n_{\alpha}^{s}=0$ and $n_{\alpha}^{s}>0$. Hereafter we call this policy the differential pricing, and superscript " $\mathrm{D}$ " is applied. When $n_{\alpha}^{s}=0$, the number of HOV users and the aggregate social cost are

$$
\begin{aligned}
\bar{t}^{D} & =\frac{2 c m K_{\alpha}}{2 c-m K_{\alpha}^{2}+(2 c(m-1)+m) K_{\alpha}} \\
S C^{D} & =\frac{c\left(2 c+m K_{\alpha}\right)}{2 c-m K_{\alpha}^{2}+(2 c(m-1)+m) K_{\alpha}}
\end{aligned}
$$

On the other hand, when $n_{\alpha}^{s}>0$,

$$
\begin{aligned}
\bar{t}^{D} & =\frac{2 c(m-1) m K_{\alpha}}{\left(c(m-1)^{2}+2 m^{2}\right) K_{\alpha}-c(m-1)^{2}} \\
S C^{D} & =\frac{c\left(2 m^{2} K_{\alpha}-c(m-1)^{2}\right)}{\left(c(m-1)^{2}+2 m^{2}\right) K_{\alpha}-c(m-1)^{2}}
\end{aligned}
$$

The condition for $n_{\alpha}^{s}>0$ to be the case is $c<\frac{m K_{\alpha}}{m-1}$.

The ranking of alternative policies in terms of $\bar{t}$ and $S C$ varies depending on parameter values. It is helpful to illustrate the results numerically. We choose the parameters as $c=0.15, m=3$. In this case, if 1 of 5 lanes is used as an HOV lane (i.e., $K_{\alpha}=0.2$ ), the share of carpooling with HOV lanes, $\bar{t}^{H O V}$, is 0.130 , which is similar to the average share in the US (0.122 in 2000). Figures 3 and 4 respectively plot the share of carpoolers and the aggregate social cost for alternative policies against the capacity of $\alpha$ lanes. In both figures, the results of three policies, HOV, HOT, and Uniform Pricing, coincide at $K_{\alpha}=0.1$, which is the critical capacity level as $c \frac{m-1}{m}=0.1$. In these figures, the result for the HOT policy is not shown for $K_{\alpha}<0.1$. This is because the results for the HOT policy in the figures are based on (17)(18) that correspond to the optimal HOT toll. As shown in Proposition 10 and Figure 2, the social cost under HOT is greater than that under HOV for $K_{\alpha}<c \frac{m-1}{m}=0.1$. Therefore the optimal HOT policy in this range of $K_{\alpha}$ is not allowing solo drivers in $\alpha$ lane. ${ }^{16}$

\footnotetext{
${ }^{16}$ It is possible to interpret the social cost under the optimal HOT policy in this case as coinciding with that under HOV policy.
} 
Figure 4 also shows that the social cost for HOV lanes becomes larger than that without the policy for $K_{\alpha}>0.159$. This result is again consistent with Proposition 9 and Figure 2.

Let us look at the results concerning pricing policies. Figure 3 shows that the share of carpoolers under uniform pricing $\left(\bar{t}^{U}\right)$ is constant and lower than that under differential pricing $\left(\bar{t}^{D}\right) \cdot \bar{t}^{D}$ is increasing with $K_{\alpha}$ for $K_{\alpha}<0.1\left(n_{\alpha}^{s}=0\right)$, while it is decreasing for $K_{\alpha}>0.1\left(n_{\alpha}^{s}>0\right)$. In the former case, as $K_{\alpha}$ is larger, carpooling is more advantageous because $\alpha$ lanes are less congested. On the other hand, when $n_{\alpha}^{s}>0$, more solo drivers use $\alpha$ lanes as $K_{\alpha}$ is larger. This crowds out carpooling. Figure 4 shows that differential pricing performs better than uniform pricing unless $K_{\alpha}$ is very small. This is because division of lanes induces sorting of heterogeneous users so that each user finds a better trade-off between congestion and the carpool organizing cost. This sorting effect is enhanced by choosing differential tolls optimally, which raises the share of carpoolers with only a small distortion from difference in congestion levels between lanes. As shown in Figure 3, the share of carpoolers under differential pricing is higher than that under uniform pricing. The uniform pricing has some advantages in that there is no distortion from difference in congestion levels, and it also gives an incentive to carpool in the form of saving toll payment. Our result shows, however, that the above stated advantages of uniform pricing are not large enough to exceed the sorting effect of differential pricing. It is observed in Figure 4 that there exists the optimal $K_{\alpha}$ for differential pricing policy that minimizes the social cost. Note that the optimal $K_{\alpha}$ is smaller than $0.1=c \frac{m-1}{m}$, where no solo driver uses lane $\alpha$, i.e., $n_{\alpha}^{s}=0$. This result is consistent with Proposition 8 .

Note that uniform pricing is inferior even to HOV policy for $K_{\alpha}<0.1$. This result is due to the effect of capacity allocation between lanes. Suppose that $K_{\alpha}$ is reduced from $K_{\alpha}=c \frac{m-1}{m}=0.1$, where $S C^{H O V}=S C^{U}$. One unit of decrease in $K_{\alpha}$ has two direct effects: a reduction in congestion on $\beta$ lanes and an increase in congestion in $\alpha$ lanes. The net of the two direct effects is positive (the social cost is decreased). A reduction in $K_{\alpha}$ also have indirect effect that is a change in distortion through change of $\bar{t}^{H O V}$. Since $\frac{\partial \bar{t}}{\partial K_{\alpha}}=\frac{c(m-1)^{2}+m}{m^{2}}>0$, the indirect effect works in the opposite direction of the direct effect, but the latter is larger than the former.

\section{Conclusion}

This paper shows that the welfare effects of HOV and HOT lanes policies vary depending on the parameters and road conditions. As already pointed out by several authors, introducing HOV policy improves the social welfare in some cases, but aggravates the situation in other cases. HOV policy encourages carpooling, thereby reducing the total traffic, but it also causes distortion from the difference in congestion levels between the two types of lanes. This distortion can be reduced by converting HOV lanes to HOT lanes: it allows solo drivers in HOV lanes. However, HOT policy has an adverse effect in that it discourages carpooling. As a result, contrary to the wide belief, converting HOV lanes to HOT lanes may reduce the social welfare under conditions that are not too unrealistic. 
We examine the alternative pricing policies: uniform and differential congestion pricing. The optimal uniform congestion pricing is not necessarily efficient, and the differential congestion pricing with HOV (HOT) lanes achieves the first-best efficient allocation if the capacity of HOV lanes is optimally chosen. Our result suggests that a better way to reduce the difference in traffic congestion in the two types of lanes is not to toll solo drivers in HOV lanes only (i.e., HOT policy) but to toll in regular lanes. Tolling in the regular lanes encourages carpooling, the number of the HOV lane users is increased, and the number of regular (now toll) lane users is decreased. This policy reduces the total number of vehicles by promoting carpooling while controlling the distortion from the difference in congestion levels between lanes.

For analytical simplicity, we assumed away some practical aspects in this paper. First, our model ignores the heterogeneity in travelers' congestion costs (time costs), as we have mentioned in the introduction. HOV lanes can be seen as a sorting mechanism to sort out travelers with different carpool organization costs. HOT lanes can further sort out travelers with different congestion costs. Since our paper does not incorporate the latter effect, the result has a bias against HOT lanes. When travelers' congestion costs are very different, introducing HOT lanes is more likely to improve the efficiency of HOV lanes. Second, we assumed that there are no additional costs in introducing HOV lanes and converting HOV lanes to HOT lanes. However, in reality, costs are incurred in painting stripes or placing traffic cones to delineate HOV lanes, and in enforcing occupancy requirements. Infrastructure and operating costs are also appreciably higher for HOT lanes than HOV lanes. A costbenefit comparison of the alternatives should take into account implementation costs as well as user costs. Third, HOT lane policy generates revenues that can be used to maintain the highway and finance the operation cost of the policy, and is politically feasible policy. These practical considerations can affect the relative merits of HOV, HOT, and the status quo. Finally, we assumed that the organization cost of a carpool, $t$, is independent of the size of the carpool, $m$. However, if carpoolers come from different families and work at different locations the cost per person will generally rise with $m$ because of the time and extra distance required to collect participants and to drop them off. We also ignored the complication that the $m$ participants in a carpool will generally have different values of $t$. This point becomes an issue particularly if we consider $m$ as a policy variable. We plan to relax these assumptions in our further work.

\section{Appendix A: Examples}

Here, we provide two examples indicating that the results in the special case $(F(t)=t$ and $C(q)=c q)$ may not be robust. The first example shows that even if $K_{\alpha}$ is reasonably small $\left(K_{\alpha}=1 / 4\right)$, introducing HOV lanes may increase every commuter's cost. Consider the following example:

Example 1. (Introducing HOV lanes may increase every commuter's cost.) Let $F(t)=0$ for all $t<\frac{17}{18}$, and $F(t)=18\left(t-\frac{17}{18}\right)$ for $t \in\left[\frac{17}{18}, 1\right]$. Let $C(q)=q, K_{\alpha}=\frac{1}{4}$ and $m=3$. The 
equilibrium condition

$$
C\left(\frac{F\left(\bar{t}^{H O V}\right)}{m K_{\alpha}}\right)+\bar{t}^{H O V}=C\left(\frac{1-F\left(\bar{t}^{H O V}\right)}{K_{\beta}}\right)
$$

can be written as

$$
\frac{18\left(\bar{t}^{H O V}-\frac{17}{18}\right)}{\frac{3}{4}}+\bar{t}^{H O V}=\frac{18\left(1-\bar{t}^{H O V}\right)}{\frac{3}{4}} .
$$

Thus, we have

$$
\bar{t}^{H O V}=\frac{20}{21}
$$

and

$$
\begin{aligned}
C_{\alpha}^{H O V}(t) & =18\left(\frac{20}{21}-\frac{17}{18}\right) \times \frac{4}{3}+t \\
& =\frac{4}{21}+t \\
\geq & \frac{4}{21}+\frac{17}{18}=\frac{143}{126}, \\
C_{\beta}^{H O V} & =24 \times \frac{1}{21}=\frac{8}{7} .
\end{aligned}
$$

Thus, all commuters pay more than 1, which can be achieved without HOV lanes (unit population with road capacity 1 makes $q=1$ ). This implies that introducing HOV lanes increases all commuters' costs, thus reducing social welfare.

The result is derived from a combination of a large value of $m$ and a small range of (relatively) high values of $t$. The latter assumption means that individuals are relatively homogeneous and carpool organization costs are substantial so that it is more likely for an HOV policy shift to affect everyone in the same direction and greatly increase the carpool organization costs. Even if we require that $F(t)>0$ for all $t>0$, it is easy to show that an introduction of HOV lanes may reduce the social welfare (though small number of commuters are better off). Thus, this example shows that if low-carpool-organization-cost type commuters are not present, and if there are large population of medium-high types, then an introduction of HOV lanes tends to reduce the social welfare.

The next example shows that converting HOV lanes to HOT lanes may increase every commuter's cost for any nontrivial toll level.

Example 2. (Converting HOV lanes to HOT lanes may increase every commuter's cost.) Let $F:[0,1] \rightarrow[0,1]$ be such that $F(t)=0$ for all $t<\frac{3}{4}$, and $F(t)=4\left(t-\frac{3}{4}\right)$ for all $t \in\left[\frac{3}{4}, 1\right]$. Let $C(q)=q, K_{\alpha}=\frac{1}{4}$ and $m=4$. The toll revenue is returned to commuters equally. Suppose that there are no HOV or HOT lanes initially. Then all commuters use regular 
lanes, and each commuter pays $C(1)=1$. We first show that in this example, introducing HOV lanes is not welfare-enhancing. Suppose first that HOV lanes are introduced. Then, the (interior solution) equilibrium choice of commuters is described by commuter type $\bar{t}^{\mathrm{HOV}}$ who is indifferent between the two routes:

$$
\frac{4\left(\bar{t}^{\mathrm{HOV}}-\frac{3}{4}\right)}{1}+\bar{t}^{\mathrm{HOV}}=\frac{4\left(1-\bar{t}^{\mathrm{HOV}}\right)}{\frac{3}{4}},
$$

or

$$
\bar{t}^{H O V}=\frac{25}{31}
$$

This implies that ${ }^{17}$

$$
\begin{aligned}
C_{\alpha}^{H O V}(t) & =4 \times\left(\frac{25}{31}-\frac{3}{4}\right)+t=\frac{7}{31}+t \\
C_{\beta}^{H O V} & =\frac{q_{\beta}}{\frac{3}{4}}=4\left(1-\frac{25}{31}\right) \times \frac{4}{3}=\frac{32}{31}>1 .
\end{aligned}
$$

Thus, the total social cost is

$$
\frac{7}{31} \times \frac{7}{31}+\int_{\frac{3}{4}}^{\frac{25}{31}} 4 t d t+\frac{32}{31} \times \frac{24}{31}=\frac{7887}{7688}>1 .
$$

Thus, introducing HOV lanes is social welfare-reducing (but not in the Pareto sense in this example: some lowest $t$ commuters are made better off by the introduction of HOV lanes).

Now, let us convert HOV lanes to HOT lanes with toll $\tau$. Toll $\tau$ needs to satisfy $\tau<\frac{25}{31}$ : otherwise, no commuter pays a toll. In order to conduct a social cost comparison, we assume that toll revenue is returned to commuters equally. The number of HOV commuters (carpooling commuters) is

$$
4\left(\tau-\frac{3}{4}\right)
$$

Given that the toll is $\tau$, we have

$$
q_{\alpha}+\tau=q_{\beta}
$$

where

$$
\begin{aligned}
q_{\alpha} & =4\left(\tau-\frac{3}{4}\right) \times \frac{1}{m K_{\alpha}}+\frac{n_{\alpha}^{s}}{K_{\alpha}}=4\left(\tau-\frac{3}{4}\right)+\frac{n_{\alpha}^{s}}{K_{\alpha}} \\
q_{\beta} & =\frac{4(1-\tau)-n_{\alpha}^{s}}{1-K_{\alpha}}
\end{aligned}
$$

\footnotetext{
${ }^{17}$ Thus, in this example, the lowest $t$ commuters $\left(t=\frac{3}{4}\right)$ are slightly better off by the introduction of HOV lanes: $C_{\alpha}\left(\frac{3}{4}\right)=\frac{7}{31}+\frac{3}{4}=\frac{119}{124}<1$. It is easy to construct an example where all commuters are worse off by the introduction of HOV lanes.
} 
Thus,

$$
4\left(\tau-\frac{3}{4}\right)+4 n_{\alpha}^{s}+\tau=\frac{16}{3}(1-\tau)-\frac{4}{3} n_{\alpha}^{s}
$$

or

$$
n_{\alpha}^{s}=\frac{25-31 \tau}{16}
$$

The toll revenue is

$$
T R(\tau)=\frac{25-31 \tau}{16} \times \tau=\frac{25}{16} \tau-\frac{31}{16} \tau^{2}
$$

and each commuter receives this amount since the population is normalized to unity. Since toll payers and regular lane users pay the same cost (indifferent)

$$
\begin{aligned}
C_{\beta}^{H O T}(\tau) & =q_{\beta}-T R(\tau) \\
& =\frac{16}{3}(1-\tau)-\frac{4}{3} n_{\alpha}^{s}-T R(\tau) \\
& =\frac{16}{3}(1-\tau)-\frac{4}{3} \times \frac{25-31 \tau}{16}-\frac{25}{16} \tau+\frac{31}{16} \tau^{2} \\
& =\frac{13}{4}-\frac{69}{16} \tau+\frac{31}{16} \tau^{2} \\
& >\frac{13}{4}-\frac{11}{4} \times \frac{25}{31}=\frac{32}{31}=C_{\beta}^{H O V}
\end{aligned}
$$

for all $\tau<\frac{25}{31}$. $\left(C_{\beta}^{H O T}(\tau)\right.$ is a convex function that is monotonically decreasing in the relevant range.) Thus, all commuters with $t \geq \tau$ are made worse off by the conversion. Moreover,

$$
\begin{aligned}
q_{\alpha} & =4\left(\tau-\frac{3}{4}\right)+\frac{n_{\alpha}^{s}}{K_{\alpha}} \\
& =4\left(\tau-\frac{3}{4}\right)+\frac{25-31 \tau}{\frac{1}{4} \times 16} \\
& =\frac{13-15 \tau}{4}
\end{aligned}
$$

and

$$
\begin{aligned}
C_{\alpha}^{H O T}(t, \tau) & =\frac{13-15 \tau}{4}+t-\operatorname{TR}(\tau) \\
& =\frac{13-15 \tau}{4}-\frac{25}{16} \tau+\frac{31}{16} \tau^{2}+t \\
& =\frac{13}{4}-\frac{85}{16} \tau+\frac{31}{16} \tau^{2}+t \\
& >\frac{13-15 \times \frac{25}{31}}{4}+t \\
& =\frac{7}{31}+t=C_{\alpha}^{\text {HOV }}(t),
\end{aligned}
$$


for all $\tau \leq \frac{25}{31}$. Thus, all commuters with $t<\tau$ are made worse off by the conversion for all $\tau<\frac{25}{31}$. This proves that in our numerical example, converting HOV lanes into HOT lanes increases all commuters' commuting costs.

This result is derived by the following mechanism. As $\tau$ goes down, fewer people carpool and the total number of cars on the road increases. With a high value of $m$, this effect is large. Thus, the regular lanes become more crowded. Although HOT policy moves some SOVs from the regular lanes to HOT lanes, a toll-paying SOV increases HOV lane traffic as well with a small value of $K_{\alpha}$.

\section{Appendix B: Proofs}

In this appendix, we collect all proofs.

\section{Proof of Proposition 1}

Suppose that commuters of type $t \leq t_{\alpha}$ use HOV lanes, and others use regular lanes. Then, type $t \leq t_{\alpha}$ pays cost

$$
C\left(\frac{F\left(t_{\alpha}\right)}{m K_{\alpha}}\right)+t=C\left(\frac{1}{m}\right)+t .
$$

If she switches to the regular lane, then she pays

$$
C\left(\frac{1-F\left(t_{\alpha}\right)}{K_{\beta}}\right)=C(1) .
$$

Thus, if the condition in the proposition is satisfied with a strict inequality (with an equality), then type $t_{\alpha}$ strictly prefers HOV lanes (is indifferent between $\mathrm{HOV}$ and regular lanes). Thus, $\bar{t}^{H O V} \geq t_{\alpha}$ holds (equality if $C(1)-C\left(\frac{1}{m}\right)=t_{\alpha}$ ). This implies that $q_{\beta} \leq 1$, and for all $t>\bar{t}^{H O V} \geq t_{\alpha}$, the payoff increases. Moreover, since type $\bar{t}^{H O V}$ is better off, we have

$$
C\left(\frac{F\left(\bar{t}^{H O V}\right)}{m K_{\alpha}}\right)+t \leq C\left(\frac{F\left(\bar{t}^{H O V}\right)}{m K_{\alpha}}\right)+\bar{t}^{H O V}=C\left(\frac{1-F\left(\bar{t}^{H O V}\right)}{K_{\beta}}\right) \leq C(1) .
$$

Since commuters with type $t<\bar{t}^{H O V}$ used to pay $C(1)$, they are all better off (equality holds only when $t=\bar{t}^{H O V}=t_{\alpha}$ ). Hence, traffic congestion in both HOV and regular lanes is eased, and commuters are better off in the Pareto sense. On the other hand, if the condition is violated, then $\bar{t}^{H O V}<t_{\alpha}$ holds. This implies that the traffic congestion in regular lanes increases. Thus, commuters with $t \geq \bar{t}^{H O V}$ would be worse off. $\square$ 


\section{Proof of Proposition 2}

The aggregate social cost with HOT lanes is written as

$$
\begin{aligned}
S C(\tau)= & C\left(\frac{F(\tau)}{m K_{\alpha}}+\frac{n_{\alpha}^{s}}{K_{\alpha}}\right) F(\tau)+\int_{0}^{\tau} t d F \\
& +n_{\alpha}^{s} C\left(\frac{F(\tau)}{m K_{\alpha}}+\frac{n_{\alpha}^{s}}{K_{\alpha}}\right) \\
& +\left(1-F(\tau)-n_{\alpha}^{s}\right) C\left(\frac{1-F(\tau)-n_{\alpha}^{s}}{K_{\beta}}\right) .
\end{aligned}
$$

The first, second, and third lines represent the aggregated costs of HOV lane users, toll payers, and regular lane users, respectively. Differentiating them with respect to $\tau$, we obtain

$$
\begin{aligned}
& S C^{\prime}(\tau) \\
= & {\left[C\left(\frac{F(\tau)}{m K_{\alpha}}+\frac{n_{\alpha}^{s}}{K_{\alpha}}\right)+C^{\prime}\left(\frac{F(\tau)}{m K_{\alpha}}+\frac{n_{\alpha}^{s}}{K_{\alpha}}\right) \frac{F(\tau)}{m K_{\alpha}}+\tau\right.} \\
& \left.-C\left(\frac{1-F(\tau)-n_{\alpha}^{s}}{K_{\beta}}\right)-C^{\prime}\left(\frac{1-F(\tau)-n_{\alpha}^{s}}{K_{\beta}}\right) \frac{\left(1-F(\tau)-n_{\alpha}^{s}\right)}{K_{\beta}}\right] F^{\prime}(\tau) \\
& +\left[C^{\prime}\left(\frac{F(\tau)}{m K_{\alpha}}+\frac{n_{\alpha}^{s}}{K_{\alpha}}\right) \frac{F(\tau)}{m K_{\alpha}}+C\left(\frac{F(\tau)}{m K_{\alpha}}+\frac{n_{\alpha}^{s}}{K_{\alpha}}\right)+C^{\prime}\left(\frac{F(\tau)}{m K_{\alpha}}+\frac{n_{\alpha}^{s}}{K_{\alpha}}\right) \frac{n_{\alpha}^{s}}{K_{\alpha}}\right. \\
& \left.-C\left(\frac{1-F(\tau)-n_{\alpha}^{s}}{K_{\beta}}\right)-\frac{\left(1-F(\tau)-n_{\alpha}^{s}\right)}{K_{\beta}} C^{\prime}\left(\frac{1-F(\tau)-n_{\alpha}^{s}}{K_{\beta}}\right)\right] \frac{d n_{\alpha}^{s}}{d \tau} \\
= & {\left[C^{\prime}\left(\frac{F(\tau)}{m K_{\alpha}}+\frac{n_{\alpha}^{s}}{K_{\alpha}}\right) \frac{F(\tau)}{m K_{\alpha}}-C^{\prime}\left(\frac{1-F(\tau)-n_{\alpha}^{s}}{K_{\beta}}\right) \frac{\left(1-F(\tau)-n_{\alpha}^{s}\right)}{K_{\beta}}\right] F^{\prime}(\tau) } \\
& +\left[-\tau+C^{\prime}\left(\frac{F(\tau)}{m K_{\alpha}}+\frac{n_{\alpha}^{s}}{K_{\alpha}}\right) \frac{F(\tau)}{m K_{\alpha}}\right. \\
& \left.+C^{\prime}\left(\frac{F(\tau)}{m K_{\alpha}}+\frac{n_{\alpha}^{s}}{K_{\alpha}}\right) \frac{n_{\alpha}^{s}}{K_{\alpha}}-\frac{\left(1-F(\tau)-n_{\alpha}^{s}\right)}{K_{\beta}} C^{\prime}\left(\frac{1-F(\tau)-n_{\alpha}^{s}}{K_{\beta}}\right)\right] \frac{d n_{\alpha}^{s}}{d \tau} .
\end{aligned}
$$

Thus,

$$
\begin{aligned}
S C^{\prime}(\tau)= & \Gamma(\tau) \times\left(F^{\prime}(\tau)+\frac{d n_{\alpha}^{s}}{d \tau}\right) \\
& -C^{\prime}\left(\frac{F(\tau)}{m K_{\alpha}}+\frac{n_{\alpha}^{s}}{K_{\alpha}}\right) \times \frac{n_{\alpha}^{s}}{K_{\alpha}} \times F^{\prime}(\tau) \\
& -\tau \times \frac{d n_{\alpha}^{s}}{d \tau},
\end{aligned}
$$


where

$$
\begin{aligned}
& \Gamma(\tau) \\
= & C^{\prime}\left(\frac{F(\tau)}{m K_{\alpha}}+\frac{n_{\alpha}^{s}}{K_{\alpha}}\right)\left(\frac{F(\tau)}{m K_{\alpha}}+\frac{n_{\alpha}^{s}}{K_{\alpha}}\right) \\
& -C^{\prime}\left(\frac{1-F(\tau)-n_{\alpha}^{s}}{K_{\beta}}\right) \frac{\left(1-F(\tau)-n_{\alpha}^{s}\right)}{K_{\beta}} .
\end{aligned}
$$

Since the congestion ratio in the regular lanes is always higher than in HOT lanes, we have

$$
\frac{F(\tau)}{m K_{\alpha}}+\frac{n_{\alpha}^{s}}{K_{\alpha}}<\frac{\left(1-F(\tau)-n_{\alpha}^{s}\right)}{K_{\beta}} .
$$

The non-decreasing and convex congestion cost function satisfies

$$
C^{\prime}\left(\frac{F(\tau)}{m K_{\alpha}}+\frac{n_{\alpha}^{s}}{K_{\alpha}}\right) \leq C^{\prime}\left(\frac{1-F(\tau)-n_{\alpha}^{s}}{K_{\beta}}\right)
$$

Therefore, we have

$$
\Gamma(\tau)<0,
$$

for all $n_{\alpha}^{s} \geq 0$. Now, recall $\frac{d n_{\alpha}^{s}}{d \tau}<0$. Thus, if the second term is zero, $S C^{\prime}(\tau)>0$ is guaranteed by having $F^{\prime}(\tau)+\frac{d n_{\alpha}^{s}}{d \tau} \leq 0$. The second term is zero when $\tau=\bar{t}^{H O V}$ holds, since it assures that $n_{\alpha}^{s}=0$ holds. Thus, evaluating the above formula at $\tau=\bar{t}^{H O V}$, ${ }^{18}$ we have

$$
\begin{aligned}
& \left.S C^{\prime}(\tau)\right|_{\tau=\bar{t}^{H O V}} \\
= & {\left[C^{\prime}\left(\frac{F(\tau)}{m K_{\alpha}}\right) \frac{F(\tau)}{m K_{\alpha}}-C^{\prime}\left(\frac{1-F(\tau)}{K_{\beta}}\right) \frac{(1-F(\tau))}{K_{\beta}}\right]\left(F^{\prime}(\tau)+\frac{d n_{\alpha}^{s}}{d \tau}\right) } \\
& -\tau \times \frac{d n_{\alpha}^{s}}{d \tau}
\end{aligned}
$$

In order to determine the sign of $\left.S C^{\prime}(\tau)\right|_{\tau=\bar{t}^{H O V}}$, we check the sign of the contents of the parenthesis:

$$
F^{\prime}(\tau)+\frac{d n_{\alpha}^{s}}{d \tau}=F^{\prime}(\tau)-\frac{\frac{C^{\prime}\left(\frac{F(\tau)}{m K_{\alpha}}\right) F^{\prime}(\tau)}{m K_{\alpha}}+\frac{C^{\prime}\left(\frac{1-F(\tau)}{K_{\beta}}\right) F^{\prime}(\tau)}{K_{\beta}}+1}{\frac{C^{\prime}\left(\frac{F(\tau)}{m K_{\alpha}}\right)}{K_{\alpha}}+\frac{C^{\prime}\left(\frac{1-F(\tau)}{K_{\beta}}\right)}{K_{\beta}}} \gtreqless 0
$$

${ }^{18}$ More precisely speaking, we take limit from below:

$$
\left.S C^{\prime}(\tau)\right|_{\tau=\bar{t}}=\lim _{\tau \uparrow t} S C^{\prime}(\tau)
$$


if and only if

$$
\begin{aligned}
& \frac{C^{\prime}\left(\frac{F(\tau)}{m K_{\alpha}}\right)}{K_{\alpha}}+\frac{C^{\prime}\left(\frac{1-F(\tau)}{K_{\beta}}\right)}{K_{\beta}} \\
\gtreqless & \frac{C^{\prime}\left(\frac{F(\tau)}{m K_{\alpha}}\right)}{m K_{\alpha}}+\frac{C^{\prime}\left(\frac{1-F(\tau)}{K_{\beta}}\right)}{K_{\beta}}+\frac{1}{F^{\prime}(\tau)} .
\end{aligned}
$$

That is,

$$
\begin{aligned}
F^{\prime}(\tau)+\frac{d n_{\alpha}^{s}}{d \tau} & \gtreqless 0 \Leftrightarrow \frac{m-1}{m K_{\alpha}} \times C^{\prime}\left(\frac{F(\tau)}{m K_{\alpha}}\right) \gtreqless \frac{1}{F^{\prime}(\tau)} \\
& \Leftrightarrow \frac{m-1}{m} \times \frac{F^{\prime}(\tau)}{K_{\alpha}} \times C^{\prime}\left(\frac{F(\tau)}{m K_{\alpha}}\right) \gtreqless 1
\end{aligned}
$$

Since $\frac{d n_{\alpha}^{s}}{d \tau}<0$, we conclude that $\left.S C^{\prime}(\tau)\right|_{\tau=\bar{t}^{H O V}}>0$ if we have

$$
\frac{m-1}{m} \times \frac{F^{\prime}(\tau)}{K_{\alpha}} \times C^{\prime}\left(\frac{F(\tau)}{m K_{\alpha}}\right)<1 .
$$

Hence, we have shown the desired result.

\section{Proof of Proposition 4}

Let $e=0$ and $e=1$ represent "solo" or "carpooling," respectively. Let $\mu_{k}^{e}: \mathbb{R}_{+} \rightarrow \mathbb{R}_{+}$be a measurable function such that $\mu_{k}^{e}(t)$ is the measure of commuters whose choice is the $k$ th lane and $e$, and whose carpool organization cost $t^{\prime}$ satisfies $t^{\prime} \leq t$. Let $\mu=\left(\mu_{k}^{e}\right)_{k \in\{1, \ldots, n\}, e \in\{0,1\}}$ be such that $\sum_{k=1}^{n} \sum_{e=0}^{1} \mu_{k}^{e}(t)=F(t)$ for all $t \in \mathbb{R}_{+}$. Thus, $\mu$ describes an assignment of commuters over $n$ lanes and their carpooling/solo decisions. Let $\mathcal{M}$ be the set of all $\mu$ functions.

Consider the social planner's optimization problem.

$$
\begin{gathered}
\min _{\mu \in \mathcal{M}} \sum_{k=1}^{n}\left\{\int C\left(n Q_{k}\right) d \mu_{k}^{0}+\int\left[C\left(n Q_{k}\right)+t\right] d \mu_{k}^{1}\right\} \\
\text { s.t. } \sum_{k=1}^{n} \sum_{e=0}^{1} \mu_{k}^{e}(t)=F(t) \\
\text { and } \mu_{k}^{e}(t) \geq 0
\end{gathered}
$$

where $Q_{k}=\int d \mu_{k}^{0}+\frac{1}{m} \int d \mu_{k}^{1}$, which is the measure of traffic on lane $k$. By the Weierstrass's Theorem, we have the optimal solution. Let $\mu^{*}$ be the solution of the above. $\mu^{*}$ satisfies the 
following conditions.

$$
\begin{aligned}
C\left(n Q_{k}\right)+C^{\prime}\left(n Q_{k}\right) n N_{k} & =\phi(t), \quad \text { if } \mu_{k}^{* 0}(t)>0 \\
C\left(n Q_{k}\right)+C^{\prime}\left(n Q_{k}\right) n N_{k} & \geq \phi(t), \quad \text { if } \mu_{k}^{* 0}(t)=0 \\
C\left(n Q_{k}\right)+t+\frac{1}{m} C^{\prime}\left(n Q_{k}\right) n N_{k} & =\phi(t), \text { if } \mu_{k}^{* 1}(t)>0 \\
C\left(n Q_{k}\right)+t+\frac{1}{m} C^{\prime}\left(n Q_{k}\right) n N_{k} & \geq \phi(t), \quad \text { if } \mu_{k}^{* 1}(t)=0
\end{aligned}
$$

where $N_{k}=\int d \mu_{k}^{0}+\int d \mu_{k}^{1}$, which is the measure of commuters using lane $k$. It is easy to see that the optimal allocation is decentralizable by levying toll per vehicle that equal to the congestion externality: $C^{\prime}\left(n Q_{k}\right) n N_{k}$ for solo drivers, and $\frac{1}{m} C^{\prime}\left(n Q_{k}\right) n N_{k}$ for car-poolers.

We can show some properties of $\mu^{*}$. Let $\left\{E^{0}\left(\mu^{*}\right), E^{1}\left(\mu^{*}\right), E^{m}\left(\mu^{*}\right)\right\}$ be a partition of $\{1, \ldots, n\}$ such that for all $k \in E^{0}\left(\mu^{*}\right), \mu_{k}^{* 1}(\infty)=0$ (a solo lane); for all $k \in E^{1}\left(\mu^{*}\right)$, $\mu_{k}^{* 0}(\infty)=0$ (a carpool lane), and for all $k \in E^{m}\left(\mu^{*}\right), \mu_{k}^{* 0}(\infty)>0$ and $\mu_{k}^{* 1}(\infty)>0$ (a mixed lane).

Suppose that there are two carpool lanes with different congestion levels. Since $C$ is strictly convex, by equating the congestion levels of the two lanes the social cost can be reduced. This is a contradiction. Similarly, in all categories, the same congestion levels must be achieved.

Second, focus on carpool and solo lanes. We claim that there exists $\bar{t} \in \mathbb{R}_{+}$such that $\sum_{k \in E^{1}} \mu_{k}^{* 0}(\bar{t})=0$ and $\sum_{k \in E^{0}} \mu_{k}^{* 1}(\bar{t})=\sum_{k \in E^{0}} \mu_{k}^{* 1}(\infty)$. Suppose not. Then, for all $\bar{t} \in \mathbb{R}_{+}$, either $\sum_{k \in E^{1}} \mu_{k}^{* 0}(\bar{t})>0$ or $\sum_{k \in E^{0}} \mu_{k}^{* 1}(\bar{t})<\sum_{k \in E^{0}} \mu_{k}^{* 1}(\infty)$. Either way, we can find $\tilde{t}$ and two sets of commuters with the same positive measures: the former is composed by car poolers whose $t$ satisfies $t>\tilde{t}$, and the latter is composed by solo drivers whose $t$ satisfies $t<\tilde{t}$. By swapping the roles of these two sets of commuters, the total social cost is decreased. This is a contradiction. Thus, there exists $\bar{t} \in \mathbb{R}_{+}$such that $\sum_{k \in E^{1}} \mu_{k}^{* 0}(\bar{t})=0$ and $\sum_{k \in E^{0}} \mu_{k}^{* 1}(\bar{t})=$ $\sum_{k \in E^{0}} \mu_{k}^{* 1}(\infty)$.

Third, similarly to the second point, among mixed lane commuters, we can say there exists $\tilde{t} \in \mathbb{R}_{+}$such that $\sum_{k \in E^{m}} \mu_{k}^{* 0}(\tilde{t})=0$ and $\sum_{k \in E^{m}} \mu_{k}^{* 1}(\tilde{t})=\sum_{k \in E^{m}} \mu_{k}^{* 1}(\infty)$.

Fourth, we claim $\bar{t}$ in the second point and $\tilde{t}$ in the third point must be the same. The proof is again by contradiction. If $\bar{t} \neq \tilde{t}$, then by swapping solo drivers whose $t$ s are lower with carpoolers whose $t$ s are higher, the total social cost can be lowered.

The above four observations prove the statement of Proposition $4 . \square$

\section{Proof of Proposition 5}


The first-order condition with respect to $n_{\alpha}^{s}(11)$ can be rearranged to

$$
\begin{aligned}
& C^{\prime}\left(\frac{F(\bar{t})}{m K_{\alpha}}+\frac{n_{\alpha}^{s}}{K_{\alpha}}\right) \frac{\left(F(\bar{t})+n_{\alpha}^{s}\right)}{K_{\alpha}}-C^{\prime}\left(\frac{1-F(\bar{t})-n_{\alpha}^{s}}{K_{\beta}}\right) \frac{\left(1-F(\bar{t})-n_{\alpha}^{s}\right)}{K_{\beta}} \\
& +C\left(\frac{F(\bar{t})}{m K_{\alpha}}+\frac{n_{\alpha}^{s}}{K_{\alpha}}\right)-C\left(\frac{1-F(\bar{t})-n_{\alpha}^{s}}{K_{\beta}}\right)-\lambda \\
= & 0 .
\end{aligned}
$$

First consider the case where $n_{\alpha}^{s}=0$. In this case, $\lambda \geq 0$ holds, and we have

$$
C^{\prime}\left(\frac{F(\bar{t})}{m K_{\alpha}}\right) \frac{F(\bar{t})}{m K_{\alpha}}+C\left(\frac{F(\bar{t})}{m K_{\alpha}}\right)+\bar{t}-C^{\prime}\left(\frac{1-F(\bar{t})}{K_{\beta}}\right) \frac{(1-F(\bar{t}))}{K_{\beta}}-C\left(\frac{1-F(\bar{t})}{K_{\beta}}\right)=0
$$

and

$$
C^{\prime}\left(\frac{F(\bar{t})}{m K_{\alpha}}\right) \frac{F(\bar{t})}{K_{\alpha}}-C^{\prime}\left(\frac{1-F(\bar{t})}{K_{\beta}}\right) \frac{(1-F(\bar{t}))}{K_{\beta}}+C\left(\frac{F(\bar{t})}{m K_{\alpha}}\right)-C\left(\frac{1-F(\bar{t})}{K_{\beta}}\right) \geq 0 .
$$

Since $C$ is a convex function and $\bar{t}>0$ holds $\left(\frac{F(\bar{t})}{m K_{\alpha}}=0\right.$, otherwise), the first-order condition with respect to $\bar{t}(10)$ implies

$$
\frac{F\left(\bar{t}^{D}\right)}{m K_{\alpha}} \leq \frac{1-F\left(\bar{t}^{D}\right)}{K_{\beta}}
$$

where $\bar{t}^{D}$ is the optimal solution. The above inequality implies that the regular lanes are more congested than HOV lanes.

We derive the pricing rule that is compatible with the optimal conditions above. In order to achieve $\bar{t}^{D}$ as the threshold type, we need to charge the following $\tau_{\beta}^{D}$ to $\beta$ lane users,

$$
\tau_{\beta}^{D}=C\left(\frac{F\left(\bar{t}^{D}\right)}{m K_{\alpha}}\right)+\bar{t}^{D}-C\left(\frac{1-F\left(\bar{t}^{D}\right)}{K_{\beta}}\right)
$$

As long as this toll is charged, all types $t>\bar{t}^{D}$ have no incentive to carpool. The first-order condition with respect to $\bar{t}^{D}(10)$ together with $\frac{F\left(\bar{t}^{D}\right)}{m K_{\alpha}} \leq \frac{1-F\left(\bar{t}^{D}\right)}{K_{\beta}}$ implies

$$
\begin{aligned}
\tau_{\beta}^{D} & =C\left(\frac{F\left(\bar{t}^{D}\right)}{m K_{\alpha}}\right)+\bar{t}^{D}-C\left(\frac{1-F\left(\bar{t}^{D}\right)}{K_{\beta}}\right) \\
& =C^{\prime}\left(\frac{1-F\left(\bar{t}^{D}\right)}{K_{\beta}}\right) \frac{\left(1-F\left(\bar{t}^{D}\right)\right)}{K_{\beta}}-C^{\prime}\left(\frac{F\left(\bar{t}^{D}\right)}{m K_{\alpha}}\right) \frac{F\left(\bar{t}^{D}\right)}{m K_{\alpha}} \\
& \geq 0 .
\end{aligned}
$$


Now, consider the case where $n_{\alpha}^{s}>0$. Since $\lambda=0$ holds, this requires

$$
\begin{aligned}
0= & C^{\prime}\left(\frac{F\left(\bar{t}^{D}\right)}{m K_{\alpha}}+\frac{n_{\alpha}^{s}}{K_{\alpha}}\right) \frac{\left(F\left(\bar{t}^{D}\right)+n_{\alpha}^{s}\right)}{K_{\alpha}}-C^{\prime}\left(\frac{1-F\left(\bar{t}^{D}\right)-n_{\alpha}^{s}}{K_{\beta}}\right) \frac{\left(1-F\left(\bar{t}^{D}\right)-n_{\alpha}^{s}\right)}{K_{\beta}} \\
& +C\left(\frac{F\left(\bar{t}^{D}\right)}{m K_{\alpha}}+\frac{n_{\alpha}^{s}}{K_{\alpha}}\right)-C\left(\frac{1-F\left(\bar{t}^{D}\right)-n_{\alpha}^{s}}{K_{\beta}}\right) \\
> & C^{\prime}\left(\frac{F\left(\bar{t}^{D}\right)}{m K_{\alpha}}+\frac{n_{\alpha}^{s}}{K_{\alpha}}\right)\left(\frac{F\left(\bar{t}^{D}\right)}{m K_{\alpha}}+\frac{n_{\alpha}^{s}}{K_{\alpha}}\right)-C^{\prime}\left(\frac{1-F\left(\bar{t}^{D}\right)-n_{\alpha}^{s}}{K_{\beta}}\right) \frac{\left(1-F\left(\bar{t}^{D}\right)-n_{\alpha}^{s}\right)}{K_{\beta}} \\
& +C\left(\frac{F\left(\bar{t}^{D}\right)}{m K_{\alpha}}+\frac{n_{\alpha}^{s}}{K_{\alpha}}\right)-C\left(\frac{1-F\left(\bar{t}^{D}\right)-n_{\alpha}^{s}}{K_{\beta}}\right) .
\end{aligned}
$$

This inequality necessarily implies

$$
\frac{F\left(\bar{t}^{D}\right)}{m K_{\alpha}}+\frac{n_{\alpha}^{s}}{K_{\alpha}}<\frac{1-F\left(\bar{t}^{D}\right)-n_{\alpha}^{s}}{K_{\beta}} .
$$

Thus, if $\alpha$ lanes are open to non-carpooling commuters, then $\beta$ lanes are more congested than $\alpha$ lanes. Now, focus on the first-order condition with respect to $\bar{t}^{D}$. Rearranging this, we can write

$$
\begin{aligned}
\tau_{\beta}^{D} & =C\left(\frac{F\left(\bar{t}^{D}\right)}{m K_{\alpha}}+\frac{n_{\alpha}^{s}}{K_{\alpha}}\right)+\bar{t}^{D}-C\left(\frac{1-F\left(\bar{t}^{D}\right)-n_{\alpha}^{s}}{K_{\beta}}\right) \\
& =C^{\prime}\left(\frac{1-F\left(\bar{t}^{D}\right)-n_{\alpha}^{s}}{K_{\beta}}\right) \frac{\left(1-F\left(\bar{t}^{D}\right)-n_{\alpha}^{s}\right)}{K_{\beta}}-C^{\prime}\left(\frac{F\left(\bar{t}^{D}\right)}{m K_{\alpha}}+\frac{n_{\alpha}^{s}}{K_{\alpha}}\right) \frac{\left(F\left(\bar{t}^{D}\right)+n_{\alpha}^{s}\right)}{m K_{\alpha}} \\
& >C^{\prime}\left(\frac{1-F\left(\bar{t}^{D}\right)-n_{\alpha}^{s}}{K_{\beta}}\right) \frac{\left(1-F\left(\bar{t}^{D}\right)-n_{\alpha}^{s}\right)}{K_{\beta}}-C^{\prime}\left(\frac{F\left(\bar{t}^{D}\right)}{m K_{\alpha}}+\frac{n_{\alpha}^{s}}{K_{\alpha}}\right)\left(\frac{F\left(\bar{t}^{D}\right)}{m K_{\alpha}}+\frac{n_{\alpha}^{s}}{K_{\alpha}}\right) \\
& >0 .
\end{aligned}
$$

The last inequality holds since we have $\frac{F\left(\bar{t}^{D}\right)}{m K_{\alpha}}+\frac{n_{\alpha}^{s}}{K_{\alpha}}<\frac{1-F\left(\bar{t}^{D}\right)-n_{\alpha}^{s}}{K_{\beta}}$.

\section{Proof of Lemma 7}

Suppose that there are multiple mixed lanes. Swapping 1 SOV and $m$ HOVs between mixed lanes does not affect the congestion levels of lanes, so the social cost are unchanged. Repeating the reallocation as above yields new solo lanes and car-pool lanes, then end up leaving one mixed lane. As the congestion level remains constant after swapping, new solo lanes are less congested than existing solo lanes. In this case, the social cost can be reduced by reallocating SOVs between new and existing solo lanes. Thus it is not optimal that there are multiple mixed lanes. 


\section{Proof of Proposition 9}

Subtracting $S C^{H O V}$ from $S C^{\emptyset}$, we have

$$
\begin{aligned}
& c-\frac{c\left(c-m K_{\alpha}\right)}{c-c K_{\alpha}+c m K_{\alpha}+m K_{\alpha}-m K_{\alpha}^{2}}+\frac{1}{2}\left(\frac{c m K_{\alpha}}{c-c K_{\alpha}+c m K_{\alpha}+m K_{\alpha}-m K_{\alpha}^{2}}\right)^{2} \\
= & \frac{c K_{\alpha}\left(c^{2}\left(2 K_{\alpha}(m-1)^{2}+2(m-1)\right)+c\left(m(3 m-4) K_{\alpha}-4(m-1) m K_{\alpha}^{2}\right)+2 m^{2} K_{\alpha}^{2}\left(K_{\alpha}-1\right)\right)}{2\left(-m K_{\alpha}^{2}+(c(m-1)+m) K_{\alpha}+c\right)^{2}}
\end{aligned}
$$

Let us define $\Omega\left(c, m, K_{\alpha}\right)$ that is the numerator of the above expression:

$$
\begin{aligned}
& \Omega\left(c, m, K_{\alpha}\right) \\
= & c K_{\alpha}\left[c^{2}\left(2 K_{\alpha}(m-1)^{2}+2(m-1)\right)+c\left(m(3 m-4) K_{\alpha}-4(m-1) m K_{\alpha}^{2}\right)+2 m^{2} K_{\alpha}^{2}\left(K_{\alpha}-1\right)\right]
\end{aligned}
$$

Function $\Omega\left(c, m, K_{\alpha}\right)$ is a cubic function of $c$ in which the coefficient of $c^{3}$ is positive. Also note that $\Omega\left(0, m, K_{\alpha}\right)=0$ and $\left.\frac{\partial \Omega}{\partial c}\right|_{c=0}=2 m^{2} K_{\alpha}^{3}\left(K_{\alpha}-1\right)<0$. Thus there exists $\widetilde{c}$ such that $\Omega\left(c, m, K_{\alpha}\right) \gtreqless 0 \Leftrightarrow c \gtreqless \widetilde{c}\left(K_{\alpha}, m\right)$, where $\widetilde{c}\left(K_{\alpha}, m\right)$ is the larger root of the quadratic equation for $c$ in the bracket of $\Omega\left(c, m, K_{\alpha}\right)$, i.e.,

$$
\begin{aligned}
& \widetilde{c}\left(K_{\alpha}, m\right) \\
= & \frac{4 K_{\alpha}^{2} m^{2}-3 K_{\alpha} m^{2}-4 K_{\alpha}^{2} m+4 K_{\alpha} m+\sqrt{-8 K_{\alpha}^{3} m^{4}+9 K_{\alpha}^{2} m^{4}+8 K_{\alpha}^{3} m^{3}-8 K_{\alpha}^{2} m^{3}}}{2\left(2 K_{\alpha} m^{2}-4 K_{\alpha} m+2 m+2 K_{\alpha}-2\right)}
\end{aligned}
$$

We have completed the proof. $\square$

\section{Proof of Proposition 10}

There are two cases: the coefficient of $\tau^{2}$ on the numerator of (15) is positive or negative, i.e.,

$$
-2 m K_{\alpha}^{2}+2(c(m-1)+m) K_{\alpha}+c(2-m) \gtreqless 0,
$$

or

$$
c \lesseqgtr \frac{2 m K_{\alpha}\left(1-K_{\alpha}\right)}{m-2-2(m-1) K_{\alpha}} .
$$

If $c<\frac{2 m K_{\alpha}\left(1-K_{\alpha}\right)}{m-2-2(m-1) K_{\alpha}}, S C^{H O T}$ is convex in $\tau$ for $\tau \in[0, \bar{t}]$, while if $c>\frac{2 m K_{\alpha}\left(1-K_{\alpha}\right)}{m-2-2(m-1) K_{\alpha}}$, $S C^{H O T}$ is concave in $\tau$ for $\tau \in[0, \bar{t}]$ (if equality, both concave and convex). The shape of $S C^{H O T}(\tau)$ can be concave if $K_{\alpha}$ is small and $m$ is large.

We first consider the case where $S C^{H O T}$ is concave in $\tau\left(c>\frac{2 m K_{\alpha}\left(1-K_{\alpha}\right)}{m-2-2(m-1) K_{\alpha}}\right)$. In this case, $\left.\frac{d S C^{H O T}(\tau)}{d \tau}\right|_{\tau=0}<0$ implies $\frac{d S C^{H O T}(\tau)}{d \tau}<0$ for all $\tau>0$. Differentiating $S C^{H O T}(\tau)$ with respect to $\tau$, we obtain

$$
\frac{d S C^{H O T}(\tau)}{d \tau}=\frac{\left(c(2-m)+2 c(m-1) K_{\alpha}+2 m K_{\alpha}\left(1-K_{\alpha}\right)\right) \tau+(1-m) c^{2}}{c m} .
$$


Applying $\tau=0$ to the above relation we have $\left.\frac{d S C^{H O T}(\tau)}{d \tau}\right|_{\tau=0}=\frac{c(1-m)}{m}<0$. Thus, $S C^{H O T}$ is monotonically decreasing in $\tau$, and $S C^{H O T}(\tau)>S C^{H O T}\left(\bar{t}^{H O V}\right)^{m}$ for all $\tau<\bar{t}^{H O V}$, and converting $\mathrm{HOV}$ lanes to HOT lanes unambiguously deteriorates the social welfare.

In contrast, if $S C^{H O T}$ is convex in $\tau\left(c<\frac{2 m K_{\alpha}\left(1-K_{\alpha}\right)}{m-2-2(m-1) K_{\alpha}}\right)$, converting HOV lanes to HOT lanes reduces the social cost for some values of toll $\tau$, if and only if $\left.\frac{d S C^{H O T}(\tau)}{d \tau}\right|_{\tau=\bar{t}^{H O V}}>0$. If $S C^{H O T}(\tau)$ is U-shaped, then there is the interior optimal toll rate $\tau^{\stackrel{d \tau}{H O T}<\bar{t}^{H O V}}$. For $\tau=\bar{t}^{H O V}$ we have

$$
\begin{aligned}
& \left.\frac{d S C^{H O T}(\tau)}{d \tau}\right|_{\tau=\bar{t}^{H O V}} \\
= & \frac{2 m^{2} K_{\alpha}^{2}\left(1-K_{\alpha}\right)-c m K_{\alpha}\left((2 m-3)-3(m-1) K_{\alpha}\right)-c^{2}(m-1)\left(K_{\alpha}(m-1)+1\right)}{c m\left((m-1) K_{\alpha}+1\right)+m^{2} K_{\alpha}\left(1-K_{\alpha}\right)}
\end{aligned}
$$

The denominator is always positive, so let us investigate the sign of the numerator. Note that the numerator is a quadratic function of $c$, and the coefficient of $c^{2}$ is negative. In this case, we obtain the condition that the numerator has a positive value as

$$
-\frac{2 m K_{\alpha}\left(1-K_{\alpha}\right)}{1+(m-1) K_{\alpha}}<c<\frac{m K_{\alpha}}{m-1}
$$

Since $c$ is supposed to have positive values, only the latter inequality should apply, i.e., $c<\frac{m K_{\alpha}}{m-1}$. And this inequality suffices as the condition for the proposition since parameters satisfying $c<\frac{m K_{\alpha}}{m-1}$ are included in the set of parameters satisfying the convexity condition $c<\frac{2 m K_{\alpha}\left(1-K_{\alpha}\right)}{m-2-2(m-1) K_{\alpha}}$

\section{References}

[1] Dahlgren, J., 1998, "High Occupancy Vehicle Lanes: Not Always More Effective Than General Purpose Lanes," Transportation Research A 32, 99-114.

[2] Fielding, G.J., and D.B. Klein, 1993, "High Occupancy/Toll Lanes: Phasing in Congestion Pricing a Lane at a Time," Policy Studies No. 170, Reason Foundation.

[3] Houde, S., E. Safirova and W. Harrington, 2007, "Washington START Transportation Model: Description and Documentation," RFF DP 07-43.

[4] O'Sullivan, A., 2007, Urban Economics (6th edition), McGraw-Hill Irwin (New York).

[5] Orki, C.K., 2001, "Carpool Lanes - An Idea Whose Time Has Come and Gone," TR News 214, 24-28.

[6] Poole, R.W. Jr., and T. Balaker, 2005, Virtual Exclusive Busways: Improving Urban Transit While Relieving Congestion, Reason Foundation Policy Study 337. 
[7] Poole, R.W. Jr., and C.K. Orski, 2000, "HOT Lanes: A Better Way to Attack Urban Highway Congestion," Regulation 23, 15-20.

[8] Reitman, D., 1991, "Endogenous Quality Differentiation in Congested Markets," Journal of Industrial Economics, 621-647.

[9] Safirova, E., Gillingham, K., Parry, I., Nelson, P., Harrington, W., and Mason, D., 2004, "Welfare and Distributional Effects of Road Pricing Schemes for Metropolitan Washington DC," in Georgina Santos (ed.), Road Pricing: Theory and Practice, Research in Transportation Economics, 9, 179-206, Elsevier.

[10] Small, K.A., 1997, "Economics and Urban Transportation Policy in the United States," Regional Science and Urban Economics 27, 671-691.

[11] Small, K.A., C. Winston, and J. Yan, 2006, "Differentiated Road Pricing, Express Lanes, and Carpools: Exploiting Heterogeneous Preferences in Policy Design," BrookingsWharton Papers on Urban Affairs, 53-96.

[12] Small, K.A., and J. Yan, 2001, "The Value of 'Value Pricing' of Roads: Second-Best Pricing and Product Differentiation," Journal of Urban Economics 49, 310-336.

[13] US Census Bureau, 2004, Journey to Work: 2000 - Census 2000 Brief, (http://www.census.gov/prod/2004pubs/c2kbr-33.pdf, accessed on June 12, 2008).

[14] Verhoef, E.K., and K.A. Small, 2004, Product Differentiation on Roads: Constrained Congestion Pricing with Heterogeneous Users, Journal of Transport Economics and Policy 38, 127-56.

[15] Yang, H., and H.-J. Huang, 1999, Carpooling and Congestion Pricing in a Multilane Highway with High-Occupancy Vehicle Lanes, Transportation Research Part A 33, 139155. 


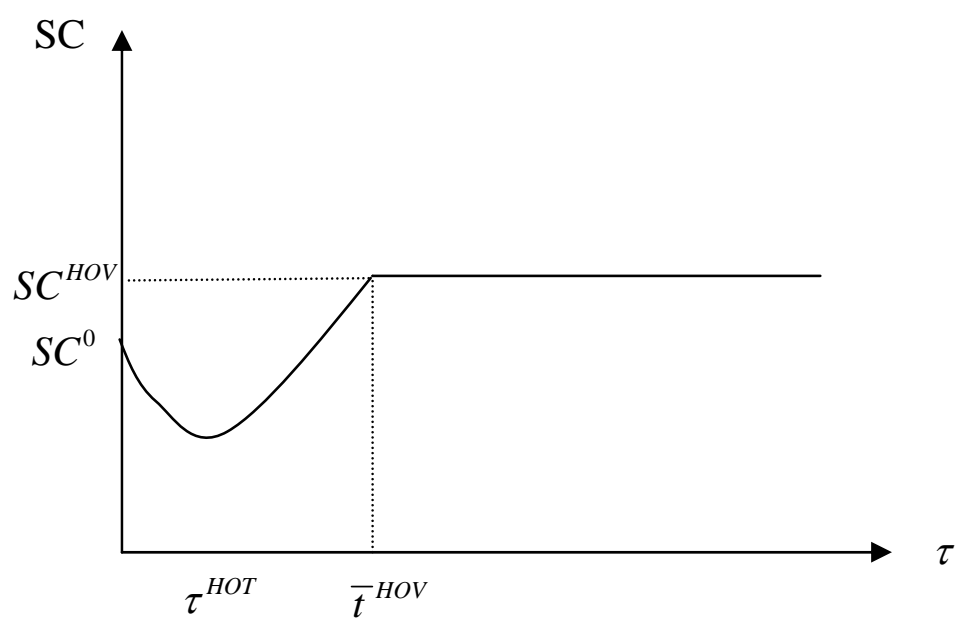

Fig.1A: Social cost may be reduced by converting HOV lane to HOT lane. Introducing HOV lane increases the social cost, i.e., $S C^{\mathrm{HOV}}>S C^{0}$

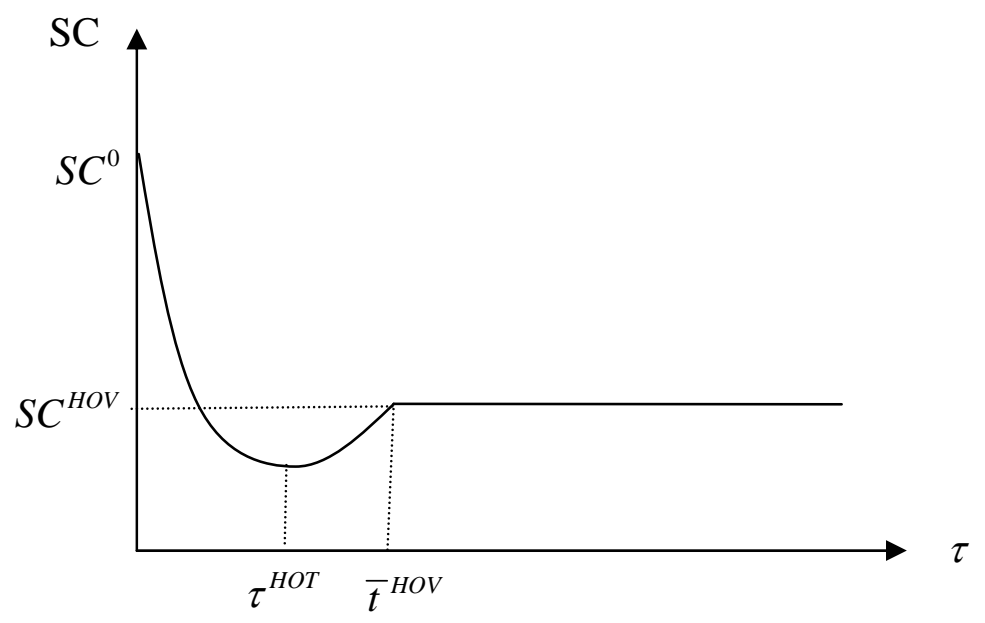

Fig.1B: Social cost may be reduced by converting HOV lane to HOT lane. Introducing HOV lane reduces the social cost, i.e., $S C^{\mathrm{HOV}}<S C^{0}$ 


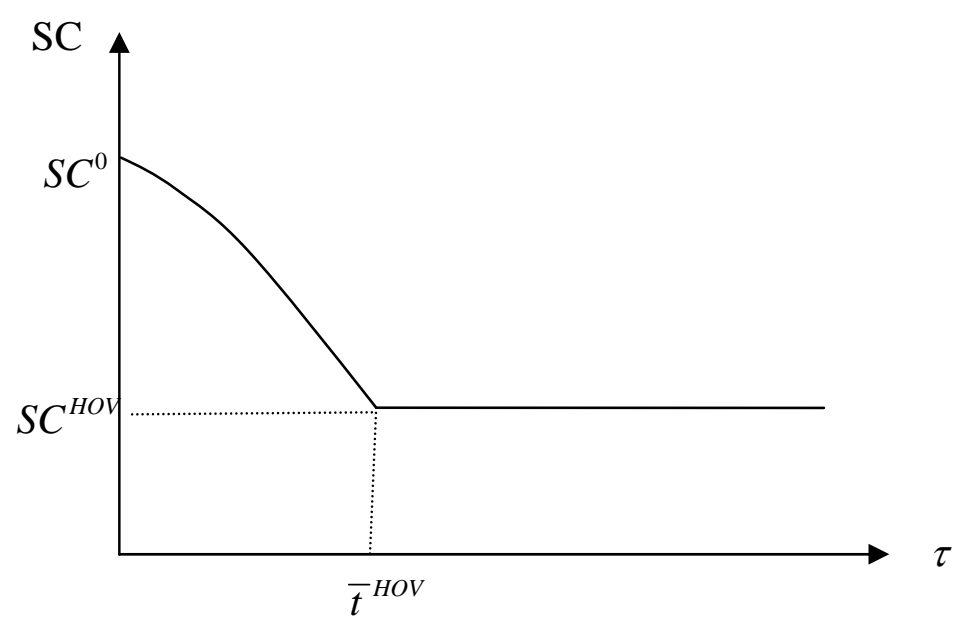

Fig.1C Converting HOV lane to HOT lane increases the social cost. Introducing HOV lane reduces the social cost, i.e., $S C^{\mathrm{HOV}}<S C^{0}$ 


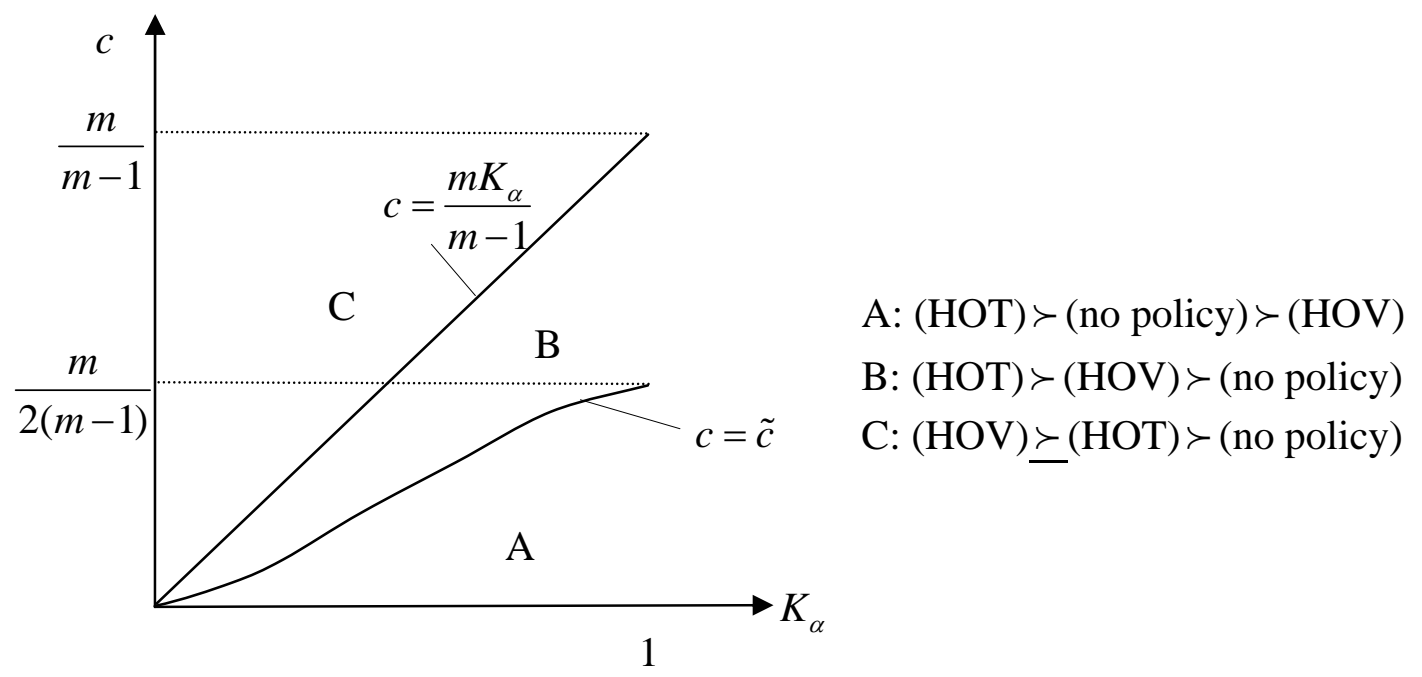

Figure 2 Parameters and welfare ranking of HOV and HOT 


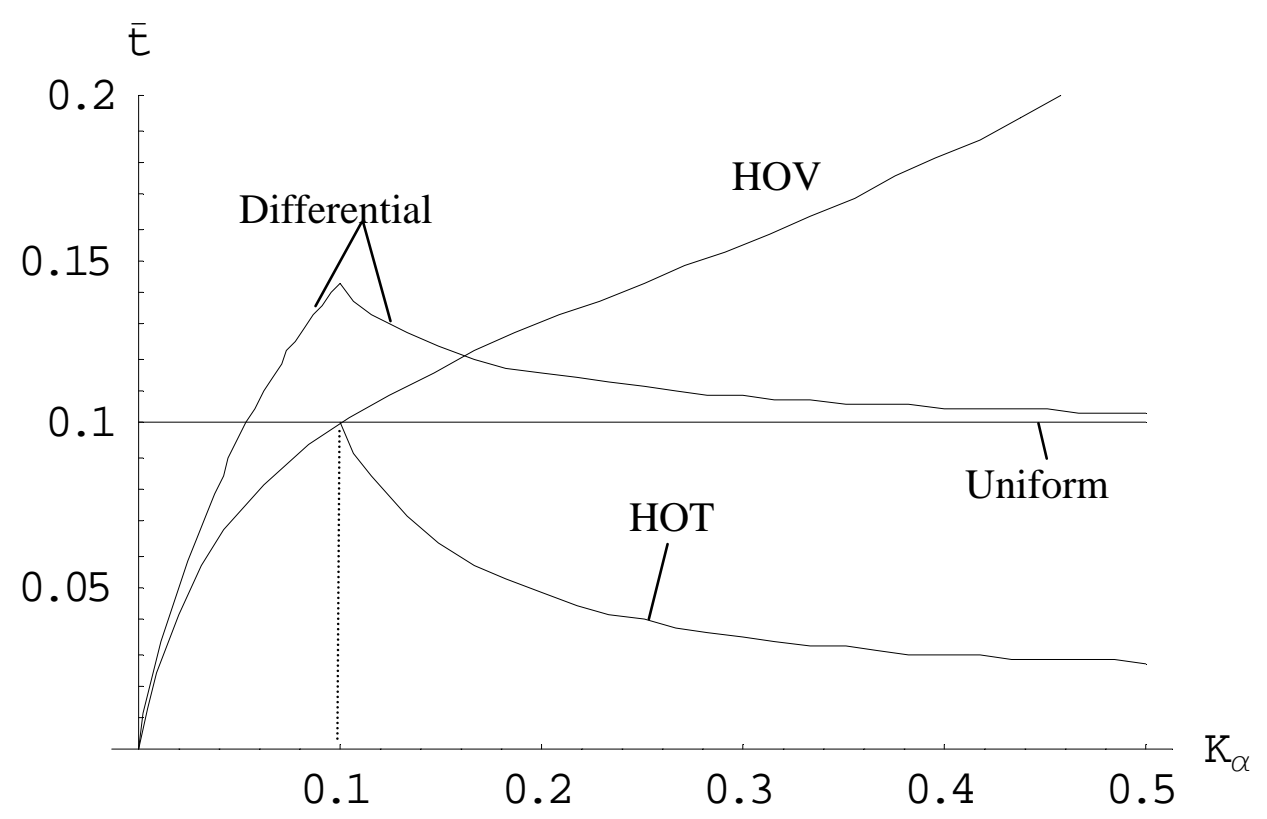

Figure 3 Shares of car-poolers under alternative policies

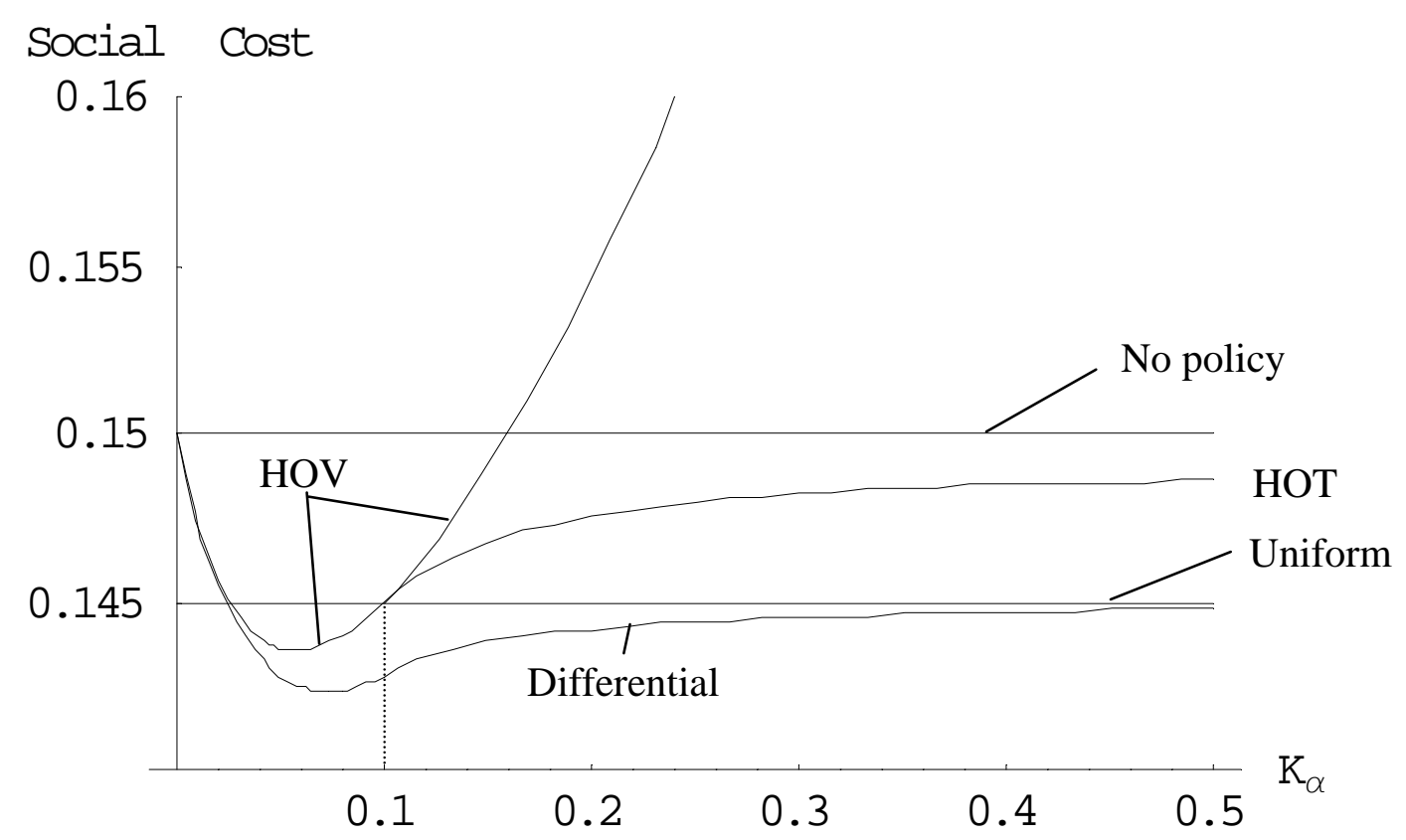

Figure 4 Aggregate social costs under alternative policies 